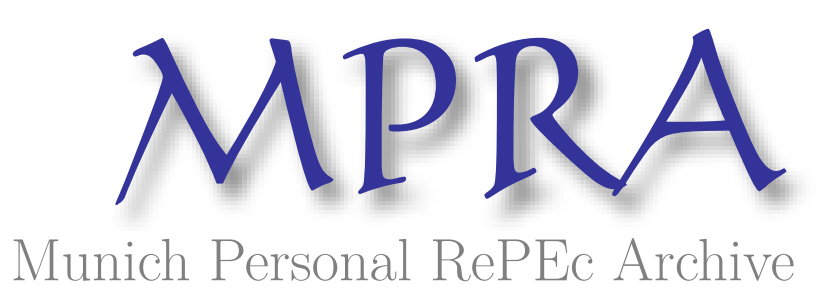

\title{
Procedural Concerns and Reciprocity
}

Sebald, Alexander

ECORE

4 June 2007

Online at https://mpra.ub.uni-muenchen.de/5066/

MPRA Paper No. 5066, posted 28 Sep 2007 UTC 


\section{ECORE}

INTERNATIONAL ASSOCIATION FOR RESEARCH AND TEACHING

Economics, Finance, Operations Research, Econometrics and Statistics

\section{ECORE DISCUSSION PAPER}

$2007 / 54$

Procedural Concerns

Alexander SEBALD 


\title{
ECORE DISCUSSION PAPER
}

\author{
Procedural Concerns*
}

\author{
Alexander Sebald ${ }^{\dagger}$
}

June 4, 2007

\begin{abstract}
Different to other scientific disciplines traditional economic theory has remained remarkably silent about procedural aspects of strategic interactions. Much to the contrast, among psychologists there is by now a broad consensus that not only expected outcomes shape human behavior, but also procedures that are used to take decisions. It is argued that procedural concerns are especially pervasive in the resolution of conflicts. In our paper we show that procedural concerns are in fact an inherent feature of the interaction of reciprocal agents. More precisely, using Dufwenberg and Kirchsteiger (2004)'s theory of sequential reciprocity we demonstrate that procedural choices determine the responsibility that people have for outcomes. The responsibility for outcomes in turn influences peoples' evaluations of intentions and, hence, subsequent reactions. Two applications are discussed to highlight the impact and importance of procedural concerns in strategic interactions.
\end{abstract}

Keywords: Psychological Games, Procedural Concerns, Reciprocity

JEL Classification: D01, C70

\section{Introduction}

Imagine a group of three friends. One of them has a free ticket for the local concert of their favorite music band. Unfortunately, however, he cannot go himself, as he has an exam the following day. As his friends love the band as much as he does, he would like to give the ticket to one of them instead. He is indifferent as to whom of the two to give it. He knows,

* I am very grateful to Martin Dufwenberg, Georg Kirchsteiger, Pierpaolo Battigalli, Estelle Cantillon, Paolo Casini and the seminar participants at ECARES/ULB and the University of Maastricht for helpful comments.

$\dagger$ Department of Economics, University of Maastricht, PO Box 616, 6200 MD Maastricht, The Netherlands, and ECARES, Universite Libre de Bruxelles. Sebald is also member of ECORE, the recently created association between CORE and ECARES. E-mail: a.sebald@algec.unimaas.nl 
however, that if one of them feels unkindly treated, he will get into a quarrel. It is easy to see that this situation bears much resemblance to the 'So long, Sucker' game analyzed e.g. by Nalebuff and Shubik (1988). A player $(A)$, i.e. the ticket holder, is driven to choose an unlucky player, i.e. the friend that does not receive the ticket, out of two players $(B)$ and $(C)$. Subsequently the unlucky player is allowed to choose an action which is either kind, i.e. not quarreling, or unkind, i.e. quarreling, towards player $(A)$. As in the 'So long, Sucker' game, it seems also here, at first sight, that the ticket holder is trapped: By choosing who gets the ticket he inevitably has to be unkind to one of his friends, creating the risk of trouble. At a second glance, however, when asked how this conflict could be resolved, one is intuitively driven to suggest that he should flip a coin to take the decision as in this way he avoids being unkind to either of them.

This example and our intuition of how to resolve the conflict effectively highlight two essential aspects of any human interaction. First, very often there are numerous ways in which decisions can be taken. On the one hand, the friend holding the ticket could decide to take the decision himself as to whom to give it, but, on the other hand, he could also let chance decide by flipping a coin. Secondly, one can easily see that decisions are inherently associated with procedures which characterize the way in which they are taken. The ticket holder, in our example, first has to decide how he wants to take the decision before he can effectively take it.

Among psychologists there is by now a broad consensus that not only expected outcomes shape human behavior, but also procedures that are used to take decisions [e.g. Thibaut and Walker (1975), Lind and Tyler (1988), Collie et al (2002), Anderson and Otto (2003) and Blader and Tyler (2003)]. It is argued that procedural concerns are especially pervasive in the resolution of conflicts. Prominent examples of conflict resolutions are to be found in the areas of workplace relations and the public acceptability of policies and laws. First, psychologists have found evidence that behavioral reactions to promotion decisions, bonus allocations, dismissals etc. strongly depend on the perceived fairness of selection procedures [e.g. Lemons and Jones (2001), Konovsky (2000), Bies and Tyler (1993), Lind, Greenberg, Scott and Welchans (2000) and Roberts and Markel (2001)]. Second, it has been shown that public compliance with policies and laws strongly depends on the perceived fairness of their enforcement procedures [e.g. Tyler (1990), Wenzel (2002), Murphy (2004), De Cremer and van Knippenberg (2003) and Tyler (2003)].

Psychologists explain the impact of procedures on human interactions with the help of attribution theory [e.g. Heider (1958), Kelley (1967), Kelley (1973), Ross and Fletcher (1985)]. Attribution theory rests on the assumption that people need to infer causes and assign responsibilities for why outcomes occur. It is argued that especially when outcomes are unfavorable and perceptions of intention are strong, there is a tendency to assign responsibility for outcomes to people. The assignment of responsibility and blame in turn has been shown to affect the occurrence and intensity of anger and aggression [Blount (1995)]. In other words, people care about others' intentions and reciprocate kind with kind and unkind with unkind behavior. As procedures explicitly influence the control that people have over final outcomes, they obviously also influence the evaluation of responsibilities and intentions. To exemplify, imagine a workplace situation in which a principal wants to promote one out of two agents. If he chooses to take the decision on who is to be promoted intransperantly behind closed doors, agents are driven to attach a high degree of responsibility for the out- 
come to the principal. His choice is interpreted as intentional, which fosters perceptions of favoritism. If, by contrast, the principal uses a transparent procedure which credibly shows that the decision is based on an unbiased criterion, i.e. a criterion which 'a priori' ensures that both agents have the same chance to be promoted, the principal is not blamed for the final outcome.

In line with attribution theory Blount (1995) experimentally showed that the responder behavior in ultimatum games is very sensitive to the way, i.e. procedure, in which a proposal is made. In her experiments proposals in the ultimatum game were either made by a proposer actively having a stake in the final outcome of the game, by a neutral third party not having any monetary stake in the final outcome or by chance. She observed that the same proposal triggered significantly lower rejection rates in case a neutral third party or chance had chosen the proposal compared to situations in which the proposal was made by a stakeholder. According to attribution theory lower rejection rates in case of neutrality of the proposer or explicit randomizations hint at the fact that responders attach a lower degree of responsibility and intentionality for outcomes to other stakeholders as they do not have any influence over proposals. In other words, the responders' willingness to punish other stakeholders seems to decrease the lower the others' influence over the final division of the pie.

Notwithstanding this experimental evidence and the fact that e.g. workplace relations play an eminent role in the economic literature, economists have remained remarkably silent so far about the impact of procedures on human behavior in strategic interactions. Only three recent economic papers have started to address the issue of procedural choices in strategic interactions [Bolton et al (2005), Trautmann (2006), Krawczyk (2007)]. In contrast to attribution theory, however, they all extend models of distributional concerns to account for the impact of procedural choices on strategic behavior. Bolton et al (2005) only present a sketch of a possible model based on the model of inequity aversion by Bolton and Ockenfels (2000). Trautmann, on the other hand, manipulates Fehr and Schmidt (1999)'s model of inequality aversion suggesting that agents' utilities depend on 'expected outcome differences' 'ex ante' as well as 'ex post' to any outcome realization. In the context of our introductory example this means that even after the flipping of a coin the ticket holder's utility depends on the 'ex ante' expected outcome difference. The expected outcome differential for his friends is lowest when flipping a coin. Hence, an inequality avers ticket holder would prefer flipping a coin to any other procedure because it ensures a zero expected outcome differential. Although Trautmann's functional form is able to accommodate the experimental finding that rejection rates in random ultimatum games are lower then in the standard ultimatum games, it can only be applied to single decision situations. It cannot be applied to more complicated strategic interactions as the calculation of expected payoffs needs expectations about the other player's play.

In contrast, our paper follows the psychologists' view. As a main result, using psychological game theory we show that procedural concerns are an inherent feature of the interaction of reciprocal agents. We first formally define the concepts of procedural game and procedure and, secondly, use the 'theory of sequential reciprocity' by Dufwenberg and Kirchsteiger (2004) to highlight the impact of procedural choices on the interaction of reciprocal agents. As will be shown, procedural choices determine the attribution of responsibilities and the evaluation of intentions. Responsibilities and intentions, in turn, determine the degree of any subsequent reciprocation. In brief, procedures are associated with explicit probability 
distributions defined over pure actions. In our concert-ticket example the two pure actions of friend $(A)$ obviously are: $i$ ) giving the ticket to friend $(B)$ and $i i)$ giving the ticket to friend $(C)$. The flipping of a coin assigns the probability $\frac{1}{2}$ to both of them. The more skewed this probability distribution is towards a certain pure action, the stronger the impression that the decision maker is intentionally aiming at this outcome. At the extreme this means, if friend $(A)$ takes the decision directly, i.e. without explicitly randomizing, to give the ticket to friend $(B)$, the unlucky friend $(C)$ assigns full responsibility and intentionality to the decision of friend $(A)$. In this situation player $(C)$ 's kindness perceptions are obviously shaped by the fact that player $(A)$ has directly chosen player $(B)$ without giving him any 'credible' chance to also get the ticket.

Dufwenberg and Kirchsteiger (2004)'s class of sequential games does not allow for different procedural choices. More precisely, it only allows for one type of procedures: procedures that imply full responsibility and intentionality. To the contrary of this, in our class of procedural games we allow for different procedural choices which then allows to analyze the impact of procedural choices on strategic interactions. To exemplify, when player $(A)$ in our introductory example decides to take his decision by flipping a coin instead of taking the decision himself both his pure actions, $i$ ) and $i i$ ), are 'ex ante' equally probable. The outcome is pure chance and, hence, no responsibility and intentionality is associated with it. As a consequence, reciprocal agents react differently to the same outcomes, i.e. choice of pure actions, depending on the procedure which has led to them.

To highlight this impact of procedural choices on the strategic interactions of reciprocal agents we analyze two applications in the final section of this paper. More precisely, we allow for different procedures in the 'So long, Sucker' game analyzed by Nalebuff and Shubik (1988) as well as Dufwenberg and Kirchsteiger (2004) and the Sequential Prisoners Dilemma also analysed by Dufwenberg and Kirchsteiger (2004). Comparing our results to their equilibrium predictions shows that the interaction of reciprocal agents is very sensitive to the availability of different procedures.

Summarizing, the organization of the paper is as follows: In the next section we formally define procedures and characterize a procedural game in which agents choose for procedures rather than actions and strategies. In the second section we point at the impact of procedures on the behavior of reciprocal agents. More precisely, we formally define reciprocity in the context of our procedural game and in this way explain the impact of procedural choices on the strategic interaction of reciprocal agents. We furthermore show that the concept of sequential reciprocity equilibria (SRE) defined by Dufwenberg and Kirchsteiger (2004) can also be applied to our class of procedural games in which agents choose for procedural strategies. Finally, as said above, two applications are discussed to highlight the impact and importance of procedural concerns in strategic interactions.

\section{Procedures}

In this section we proceed in two steps. First, we intuitively sketch our argument with the help of two examples. In a second step we $i$ ) formally define the concept of procedures and ii) fully characterize our class of procedural games in which agents do not choose actions and strategies, as usually assumed in game theory, but procedures. This class of multi-stage 
games in which agents choose procedures is thenceforth used in the subsequent sections to analyze the impact of procedural choices on the strategic interaction of reciprocal agents.

As a starting point consider games $\Gamma_{1}$ and $\Gamma_{2}$ in Figure 1 and 2:

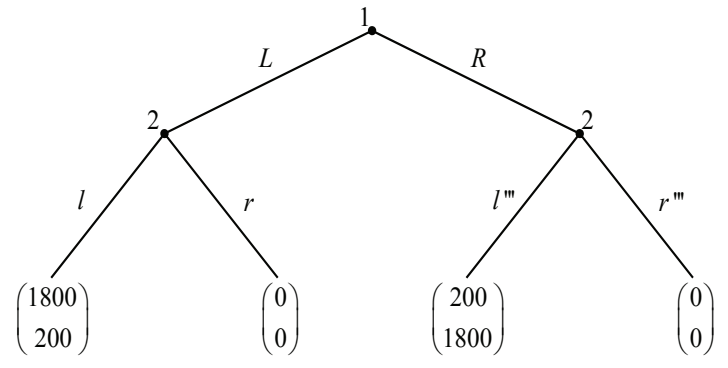

Figure 1: Game $\Gamma_{1}$

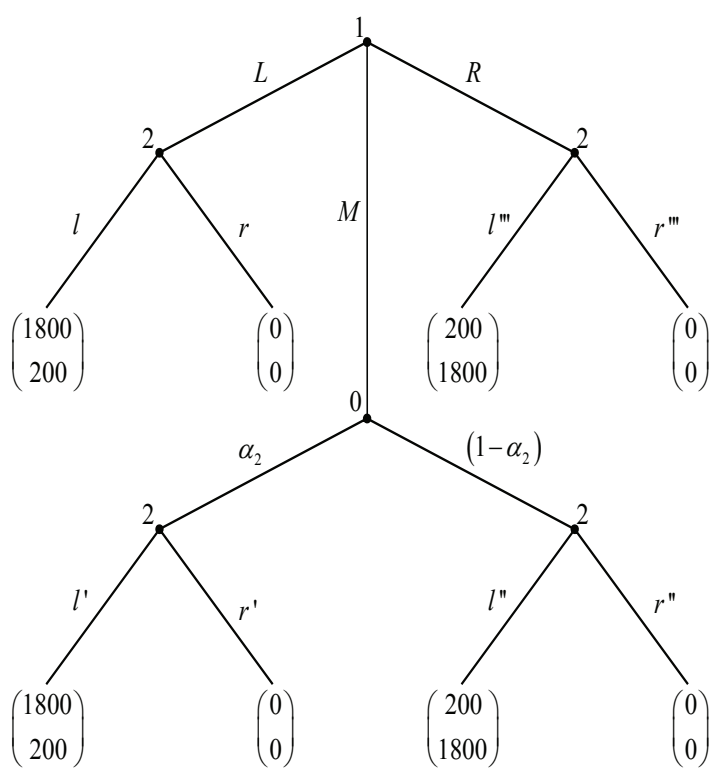

Figure 2: Game $\Gamma_{2}$

The sole difference between games $\Gamma_{1}$ and $\Gamma_{2}$ is that in $\Gamma_{2}$ player 1 can choose $(M)$ on top of his pure actions $(L)$ and $(R)$. Player 1's pure action $(M)$, however, is nothing else than choosing an explicit randomization device, (0), assigning probabilities $\alpha_{2}$ and $\left(1-\alpha_{2}\right)$ to his pure actions $(L)$ and $(R)$ respectively. 'Flipping a coin' or 'throwing a dice' constitute explicit randomization devices, for example. 'Flipping a coin' assigns the probability $\frac{1}{2}$ to both pure actions $(L)$ and $(R)$. 'Throwing a dice', on the other hand, leads to $\alpha_{2}=\frac{5}{6}$ and $\left(1-\alpha_{2}\right)=\frac{1}{6}$, if, for example, $(L)$ is chosen, whenever numbers 1 to 5 come up, and $(R)$ is chosen, if 6 appears. Obviously, 'flipping a coin' and 'throwing a dice' are but two credible ways in which a decision can be taken. In reality one usually disposes of many different ways. Nevertheless the two examples suffice to show how different ways, or in our words explicit randomization devices, are associated with differing explicit probability distributions with which an action is indirectly chosen by chance.

But not only choices like $(M)$ can be characterized as choices for explicit randomization devices. Taking the thought about the credible ways and the differing explicit probability distributions to the extreme shows that also pure actions like $(L)$ and $(R)$ can equally be defined as choices for explicit randomization mechanisms. Imagine, for example, that player 1 in $\Gamma_{1}$ and $\Gamma_{2}$ chooses for his pure actions $(L)$. This is equivalent to saying that player 1 chooses for chance to take the decision between $(L)$ and $(R)$ assigning probability 1 to his pure action $(L)$. Hence, although $(L)$ represents a pure action, it can nevertheless be reinterpreted in a way in which the decision is indirectly taken by chance randomizing with a degenerated probability distribution over the set $\{(L),(R)\}$.

This shows that in our two examples, $\Gamma_{1}$ and $\Gamma_{2}$, any choice for a pure actions, i.e. $(L)$ and $(R)$, and any choice for an explicit randomization mechanism, i.e. $(M)$, can likewise 
be reinterpreted as a choice for an explicit randomization device through which the actual decision is subsequently taken by chance. Consider, for example, game $\Gamma_{3}$ in Figure 3, which is a restatement of game $\Gamma_{2}$ in the spirit of this intuition:

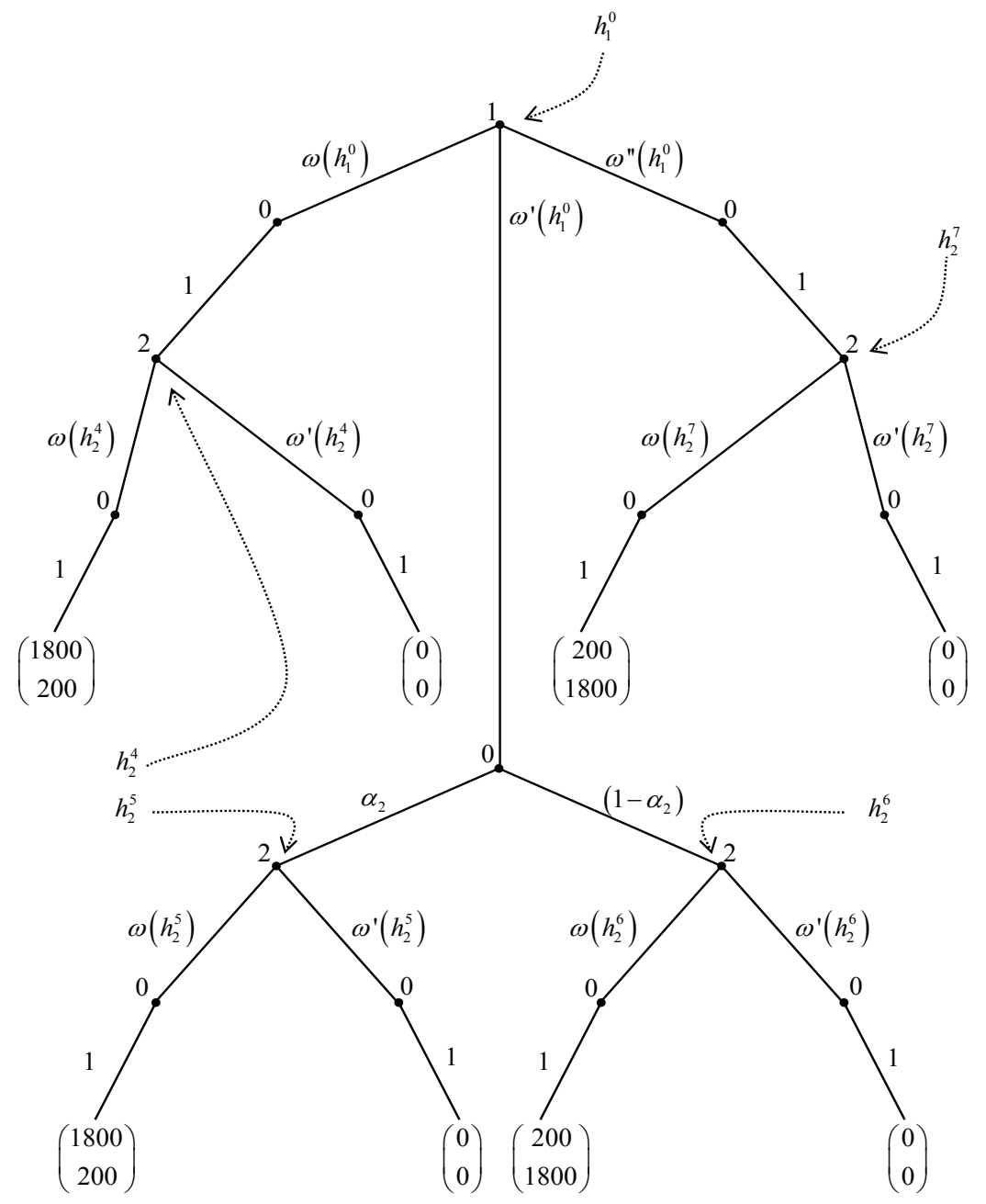

Figure 3: Game $\Gamma_{3}$

As one can see, in $\Gamma_{3}$ we reformulate all strategic choices of game $\Gamma_{2}$ into choices for explicit randomization mechanisms, i.e. chance or player 0 , through which decisions are subsequently taken. In game $\Gamma_{2}$ player 1 can decide between $(L),(M)$ and $(R)$, and player 2 can decide between $(l)$ and $(r),\left(l^{\prime}\right)$ and $\left(r^{\prime}\right),\left(l^{\prime \prime}\right)$ and $\left(r^{\prime \prime}\right)$ or $\left(l^{\prime \prime \prime}\right)$ and $\left(r^{\prime \prime \prime}\right)$ depending on player 1 's choice. Equivalently, in game $\Gamma_{3}$ player 1, for example, has to decide between the explicit randomization devices $\omega\left(h_{1}^{0}\right), \omega^{\prime}\left(h_{1}^{0}\right)$ and $\omega^{\prime \prime}\left(h_{1}^{0}\right)$ in the initial history $h_{1}^{0}$. First, by choosing $\omega\left(h_{1}^{0}\right)$ he can decide to let chance take the decision between $(L)$ and $(R)$ assigning probability 1 to $(L)$. Second, by choosing $\omega^{\prime}\left(h_{1}^{0}\right)$ he can decide to let chance take the decision between $(L)$ and $(R)$ assigning probability $\alpha_{2}$ to $(L)$ and $\left(1-\alpha_{2}\right)$ to $(R)$. Finally, by choosing $\omega^{\prime \prime}\left(h_{1}^{0}\right)$ he can decide to let chance take the decision between $(L)$ and $(R)$ assigning probability 1 to $(R)$. In all these three cases player 1 only determines how chance subsequently takes 
the decision, rather then taking the decision himself. Hence, notwithstanding the formal equivalence between games $\Gamma_{2}$ and $\Gamma_{3}$, an interpretive difference exists. Choosing for an explicit randomization mechanism implies that players do not take decisions themselves. They merely determine how decisions are taken by chance. In other words, players decide about the procedures which are used to take decisions. The example in Figure 3, thus, uncovers that strategic decision making is not only about choosing actions but also about how actions are chosen. For this reason we call game $\Gamma_{3}$ a procedural game.

This brings us to a more formal definition of our class of procedural games. Formally, let the set of players be $\mathcal{N}=\{0,1, \ldots, N\}$ where 0 denotes the uninterested player chance. Denote as $\mathcal{H}$, with the empty sequence $\varnothing \in \mathcal{H}$, the finite set of histories, $h$, and $\mathcal{X}$ the finite set of decision nodes $x$, such that $h^{x}$ is the sequence of decisions on the path to the decision node $x$. The player function, $\mathcal{C}$, assigns to each nonterminal history $h^{x} \in \mathcal{H}$ a member $i \in \mathcal{N}$ who moves after that history $h^{x}$. Therefore, let $h_{i}^{x}$ be the history $h$ on the path to the decision node $x$ which is controlled by player $i \in \mathcal{N}$ and $\mathcal{H}_{i}$ the set of all histories after which player $i$ has to move throughout the game. At each history, $h_{i}^{x}$, after which player $i \in \mathcal{N} \backslash\{0\}$ has to move, he disposes of a nonempty finite set of pure actions $\mathcal{A}\left(h_{i}^{x}\right)$ and a finite set of explicit randomization devices, $\Omega\left(h_{i}^{x}\right)$, through which he can choose an action from $\mathcal{A}\left(h_{i}^{x}\right)$. As already suggested in example $\Gamma_{3}$ players in our procedural games do not choose actions $a \in \mathcal{A}\left(h_{i}^{x}\right)$ directly, but choose explicit randomization mechanisms, denoted $\omega\left(h_{i}^{x}\right) \in \Omega\left(h_{i}^{x}\right)$, through which a decision is indirectly taken by chance. The choice for a specific explicit randomization device, $\omega\left(h_{i}^{x}\right)$, in history $h_{i}^{x}$ by player $i \in \mathcal{N} \backslash\{0\}$ leads to a specific decision node $v \in \mathcal{X}$ defined by $h_{0}^{v}$ in which chance takes the actual decision using the explicit probability distribution $\rho\left(\omega\left(h_{i}^{x}\right)\right)$ associated with $\omega\left(h_{i}^{x}\right)$ defined on $\mathcal{A}\left(h_{0}^{v}\right)$, with $\mathcal{A}\left(h_{0}^{v}\right)=\mathcal{A}\left(h_{i}^{x}\right)$. Hence, the choice for a pure action $a$ (e.g. $(L)$ in $\left.\Gamma_{2}\right)$, for example, translates in our procedural game into a choice for an explicit randomization mechanisms, $\omega\left(h_{i}^{x}\right)$, that is associated with a degenerated probability distribution $\rho\left(\omega\left(h_{i}^{x}\right)\right)$ which assigns probability 1 to the pure action $a$ in the set of possible actions $\mathcal{A}\left(h_{0}^{v}\right)=\mathcal{A}\left(h_{i}^{x}\right)$. The choice for an explicit randomization (e.g. $(M)$ in $\Gamma_{2}$ ), on the other hand, is a choice for an explicit randomization mechanism, $\omega^{\prime}\left(h_{i}^{x}\right)$, that is associated with a non-degenerated probability distribution $\rho\left(\omega^{\prime}\left(h_{i}^{x}\right)\right)$ defined on $\mathcal{A}\left(h_{0}^{v}\right)=\mathcal{A}\left(h_{i}^{x}\right)$. As said before, the set of player $i$ 's degenerated as well as non-degenerated explicit randomization mechanisms in any history $h_{i}^{x}$ is $\Omega\left(h_{i}^{x}\right)$. The associated set of explicit probability distributions is furthermore denoted as $\mathcal{P}\left(h_{i}^{x}\right)$, where $\mathcal{P}\left(h_{i}^{x}\right)=\left\{\rho\left(\omega\left(h_{i}^{x}\right)\right) \mid \omega\left(h_{i}^{x}\right) \in \Omega\left(h_{i}^{x}\right)\right\}$. It can easily be seen that the minimum number of explicit randomization mechanisms that a player can decide between in any history $h_{i}^{x}$ in our procedural game equals the number of pure actions that he has in the traditional extensive form representation.

As said before, by choosing for randomization devices players do not take decisions directly but only determine how chance subsequently takes them. Intuitively, as players only decide on how the decisions are subsequently taken, they only decide on the procedure, which is used to take a decision.

This brings us to a formal definition of procedures:

Definition 1 A procedure, $\omega\left(h_{i}^{x}\right) \in \Omega\left(h_{i}^{x}\right)$, for player $i \in \mathcal{N} \backslash\{0\}$ in history $h_{i}^{x} \in \mathcal{H}_{i}$ is a tuple:

$$
\left\langle\rho\left(\omega\left(h_{i}^{x}\right)\right), \mathcal{A}\left(h_{0}^{v}\right)\right\rangle,
$$


where:

1. $\rho\left(\omega\left(h_{i}^{x}\right)\right)$ is the explicit probability distribution associated with $\omega\left(h_{i}^{x}\right)$ defined on $\mathcal{A}\left(h_{0}^{v}\right)$

2. $\mathcal{A}\left(h_{0}^{v}\right)=\mathcal{A}\left(h_{i}^{x}\right)$, and

3. $h_{0}^{v}$ directly succeeds $h_{i}^{x}$.

In example $\Gamma_{3}$ procedures are used to choose for pure actions. We do not exclude, however, the possibility of procedures that choose between procedures and procedures that choose between procedures that choose between procedures etc. Procedures, $\omega\left(h_{i}^{x}\right) \in \Omega\left(h_{i}^{x}\right)$, rather have to be understood as reduced procedures. At any history $h_{i}^{x}$ the explicit probability distribution associated with a reduced procedure, $\rho\left(\omega\left(h_{i}^{x}\right)\right) \in \mathcal{P}\left(h_{i}^{x}\right)$, basically subsumes the probability distributions of procedures of all levels into one explicit distribution defined on $\mathcal{A}\left(h_{i}^{x}\right)$. It is assumed that all players learn the outcome of a reduced procedure directly after its realization.

We denote a collection of procedures for any player $i \in \mathcal{N} \backslash\{0\}$ that specifies a procedure for each history after which player $i$ moves a procedural strategy, $\omega_{i}$. A behavioral procedural strategy, $m_{i} \in \mathcal{M}_{i}$, of player $i$, on the other hand, has to be understood as an implicit randomization at each history $h_{i}^{x} \in \mathcal{H}_{i}$ over the set of possible procedures $\Omega\left(h_{i}^{x}\right)$. Note, procedural strategies, $\omega_{i} \in \Omega_{i}$, and behavioral procedural strategies, $m_{i} \in \mathcal{M}_{i}$, in our class of procedural games are respectively the analogue to pure strategies and mixed strategies in the traditional extensive form representation. We assume throughout that players choose for behavioral procedural strategies.

Given a behavioral procedural strategy, $m_{i}$, for each player $i \in \mathcal{N} \backslash\{0\}$ and the commonly known system of probability distributions, $\mathcal{P}=\cup_{i \in \mathcal{N} \backslash\{0\}} \mathcal{P}_{i}$, where $\mathcal{P}_{i}=\cup_{h_{i}^{x} \in \mathcal{H}_{i}} \mathcal{P}\left(h_{i}^{x}\right)$, we can compute a probability distribution over endnodes, $z \in \mathcal{Z}$. By assigning payoffs to endnodes, we can derive an expected payoff function, $\pi_{i}: \mathcal{Z} \times \mathcal{P} \rightarrow \Re$, for every player $i \in \mathcal{N} \backslash\{0\}$ which depends on what behavioral procedural profile, $m$ in $\mathcal{M}$, where $\mathcal{M}=\times_{\mathcal{N} \backslash\{0\}} \mathcal{M}_{i}$, is played. In what follows we will assume that payoffs are material payoffs like money or any other measurable quantity of some good.

Summarizing, a procedural game is a tuple:

$$
\Gamma=\left\langle\mathcal{N}, \mathcal{M}, \mathcal{P},\left(\pi_{i}: \mathcal{Z} \times \mathcal{P} \rightarrow \Re\right)_{\mathcal{N} \backslash\{0\}}\right\rangle
$$

This concludes the definition of procedures and the characterization of the class of procedural games which is the basis of our subsequent analysis. Starting from two simple examples, i.e. $\Gamma_{1}$ and $\Gamma_{2}$, we have formalized the idea that players choose for procedures rather than actions. In the remainder of the paper we use this class of procedural games in order to isolate the impact of procedures on strategic behavior. More precisely, the following section uses this characterization of procedural games to analyze the impact of procedural choices on the interaction of reciprocal agents.

\section{Procedural choices and reciprocity}

It is easy to see that if agents are only interested in their own expected material payoff, they would always behave the same in histories representing starting points of identical 
subgames. Looking again at game $\Gamma_{3}$ in Figure 3, for example, this means that players would react the same in histories $h_{2}^{4}$ or $h_{2}^{5}$. However, experimental evidence contradicts this. For example, in ultimatum games rejection rates for the same proposal significantly decrease if proposals are made by a random draw [Blount (1995) and Bolton et al (2005)]. In other words proposer behaviors in ultimatum games significantly depend on how a certain proposal has come about. Psychologists have termed this dependence procedural fairness or procedural concerns and explain the observed behavior with the help of attribution theory. According to attribution theory agents behave reciprocally and evaluate the (un)kindness of themselves and others taking into consideration their as well as the others' possible influence on (expected) outcomes. The less influence people have over outcomes at the time of their decision the less they are held responsible for it. Therefore, in order to demonstrate how procedural concerns can theoretically be reconciled with economic theory, we broaden the behavioral presumption in this section by assuming that agents are reciprocal. This means we formally define reciprocity in the context of our procedural game and show how it can explain the aforementioned evidence on procedural concerns.

Generally speaking, reciprocity means that agents do not only care about their own material payoff but also about the intentions of others [e.g. Rabin (1993), Dufwenberg and Kirchsteiger (2004) and Falk and Fischbacher (2006)]. They act kindly or unkindly depending on whether others are kind or unkind to them. Before we can more formally characterize the motivation of reciprocal agents and precisely define kindness and perceived kindness, however, it is necessary to highlight four theoretical peculiarities: kindness and perceived kindness of any player towards/from any other player $i$ ) cannot be measured directly, ii) might change after different histories of a game, iii) should be unaffected by inefficient procedural strategies and $i v$ ) realizations of the moves of chance.

i) Kindness and perceived kindness cannot be measured directly as they depend on each player's procedural strategies, beliefs about the others' procedural strategies and beliefs about the others' beliefs. Therefore, to model kindness we assume that every player holds a belief over the behavioral procedural strategies as well as a belief over the other players' beliefs. In the spirit of Dufwenberg and Kirchsteiger (2004) we model beliefs as behavioral procedural strategies, $m_{i} \in \mathcal{M}_{i}, \forall i \in \mathcal{N} \backslash\{0\}$. However, in order to avoid confusion we introduce a separate notation for beliefs. Let $\mathcal{B}_{i j}=\mathcal{M}_{j}, \forall i, j \in \mathcal{N} \backslash\{0\}$ be the set of possible beliefs of player $i$ about the behavioral procedural strategy of player $j$ (i.e. first-order belief). Furthermore let $\mathcal{C}_{i j q}=\mathcal{B}_{j q}=\mathcal{M}_{q}, \forall i, j, q \in \mathcal{N} \backslash\{0\}$ be the set of possible beliefs of player $i$ about the belief of player $j$ about the behavioral procedural strategy of player $q \neq j$ (i.e. second-order belief). Obviously, players do not have beliefs about the moves of the player chance. They do know, however, the explicit probability distributions associated with them. Therefore, let $(a)_{h^{x}}$ denote the collection of all passed realizations of moves of chance on the path up to history $h^{x}$.

ii) Players are assumed to have initial first- and second-order beliefs about the other players. As the game unravels these beliefs might change, however. In order to capture this it is important to keep track of how each player's behavior, beliefs, kindness and kindness perceptions differ across histories. We do this by updating behavioral procedural strategies as well as first- and second-order beliefs at each history that players control. In the spirit of Dufwenberg and Kirchsteiger (2004) we therefore formally define an (updated) behavioral procedural strategy as: 
Definition 2 Let $m_{i} \in \mathcal{M}_{i}$ and $h_{i}^{x} \in \mathcal{H}_{i}$, let $m_{i}\left(h_{i}^{x}\right) \in \mathcal{M}_{i}$ be the (updated) behavioral procedural strategy that prescribes the same procedural choices as $m_{i}$ except for the procedural choices of player $i$ on the path to $h_{i}^{x}$ which are made with probability 1.

In correspondence with the collection of passed realizations of the moves of chance, $(a)_{h_{i}^{x}}$, the collection of passed procedural choices of player $i$ on the path to $h_{i}^{x}$ is denoted $\left(\omega_{i}\right)_{h_{i}^{x}}$. Hence, the updated behavioral procedural strategy $m_{i}\left(h_{i}^{x}\right)$ is identical to $\left(\omega_{i}\right)_{h_{i}^{x}}$ on the path to history $h_{i}^{x}$ and identical to the initial behavioral procedural strategy, $m_{i}$, in all other histories. To exemplify consider again game $\Gamma_{3}$ in Figure 3. Let player 2's initial behavioral procedural strategy $m_{2}$ be an implicit randomization over his set of pure procedures at each history that he controls. Player 2 moves after history $h_{2}^{5}$, which means that the implicit randomization prescribed by his initial behavioral procedural strategy over his pure procedural choices, $\omega\left(h_{2}^{5}\right)$ and $\omega^{\prime}\left(h_{2}^{5}\right)$, leads to some realization. Following this his updated behavioral procedural strategy becomes such that the implicit randomization at $h_{2}^{5}$ is substituted by its realization, but all other procedural choices at histories not reached remain the same. The updating of beliefs is assumed to work in an analogous fashion. Let, for example, player 2's initial belief about player 1's behavioral procedural strategy be $b_{21}=\left(\omega\left(h_{1}^{0}\right)\right)$. If later on he finds himself in history $h_{2}^{5}$ in game $\Gamma_{3}$, his updated belief about player 1's behavioral procedural strategy becomes $b_{21}\left(h_{2}^{5}\right)=\left(\omega^{\prime}\left(h_{1}^{0}\right)\right)$, where $b_{21}\left(h_{2}^{5}\right)$ is player 2's updated firstorder belief in history $h_{2}^{5}$ about player 1's behavioral procedural strategy. This shows that, parallel to the definition of $m_{i}\left(h_{i}^{x}\right)$, the updated first order belief $b_{i j}\left(h_{i}^{x}\right)$ is identical to the passed procedural choices of player $j$ on the path to $h_{i}^{x},\left(\omega_{j}\right)_{h_{i}^{x}}$, and identical to the initial belief, $b_{i j}$, in all other histories.

A remark on mixed strategies and procedures. The concept of psychological games was first introduced by Geanakoplos et al.(1989). In their seminal work Geanakoplos et al.(1989) only allow for initial beliefs to enter utility functions. Dufwenberg and Kirchsteiger (2004) and more recently Battigalli and Dufwenberg (2005) have shown, however, that in modeling, for example, reciprocity in a sequential setting unreasonable conclusions might be drawn if utility functions only depend on initial beliefs. ${ }^{1}$ They show that it is necessary to keep track of how beliefs change as play unravels. Two areas in which the updating of beliefs needs some further explanation are mixed strategies and beliefs in mixed strategies. Dufwenberg and Kirchsteiger (2004) allow for mixed strategies and also allow players to hold beliefs in mixed strategies. Mixed strategies in their setting should be interpreted in terms of frequencies with which pure choices are made in a 'population'. This interpretation then explains why players that possibly hold mixed beliefs about the action of some other player update their beliefs (as soon as they learn his choice) as if he had chosen his actions with probability 1, i.e intentionally. Procedures, in comparison to that, might assign probabilities to pure actions in equivalence to mixed strategies. As they are observable, however, players do not update their beliefs after learning their outcome. If a player, for example, uses the flip of a coin to take a decision, this is observed by other players. This observability and the fact that probabilities connected to procedures are common knowledge implies that procedural choices represent perfect signals about intentions. Consequently, player's beliefs are updated taking

\footnotetext{
${ }^{1}$ For a more detailed discussion of this issue refer to Battigalli and Dufwenberg (2005) and Dufwenberg and Kirchsteiger (2004).
} 
into account the degree with which specific outcomes are intentionally aimed at. Therefore, in contrast to Dufwenberg and Kirchsteiger (2004), in our setting players update their beliefs according to the observed procedural choices that players make.

iii) For the same reason as in Dufwenberg and Kirchsteiger (2004) we restrict our attention to the set of efficient procedural strategies, $\mathcal{E}_{i}$. The set of efficient procedural strategies, $\mathcal{E}_{i}$, is defined as:

$$
\begin{aligned}
\mathcal{E}_{i}= & \left\{m_{i} \in \mathcal{M}_{i} \mid \text { there exists no } m_{i}^{\prime} \in \mathcal{M}_{i} \text { such that for all } h_{i}^{x} \in \mathcal{H}_{i},\left(m_{j}\right)_{j \neq i} \in \Pi_{j \neq i} \mathcal{M}_{j},\right. \\
& q \in \mathcal{N} \backslash\{0\} \text { it holds that } \pi_{q}\left(m_{i}^{\prime}\left(h_{i}^{x}\right),\left(m_{j}\left(h_{i}^{x}\right)\right)_{j \neq i}\right) \geq \pi_{q}\left(m_{i}\left(h_{i}^{x}\right),\left(m_{j}\left(h_{i}^{x}\right)\right)_{j \neq i}\right) \\
& \text { with strict inequality for some } \left.\left(h,\left(m_{j}\left(h_{i}^{x}\right)\right)_{j \neq i}, q\right)\right\} .
\end{aligned}
$$

Strategic choices are inefficient if there exists at least one other choice which conditional on any history of play and subsequent choices by the others provides no lower material payoff for any player, and a higher expected material payoff for some player for some history of play and subsequent choices by the others. In other words any behavioral procedural strategy is inefficient if it involves 'wasteful play' following some history, $h_{i}^{x} \in \mathcal{H}_{i}$. As also pointed out by Dufwenberg and Kirchsteiger (2004), it is unreasonable to let kindness and perceived kindness be influenced by strategies or, in our context, procedural strategies that imply 'wasteful play'. More precisely, the fact that 'wasteful play' is possible should be irrelevant for drawing conclusions regarding the kindness of the others' 'efficient' choices. ${ }^{2}$

$i v$ ) As said above, kindness and perceived kindness should also be unaffected by the realizations of the move of chance. Intuitively this captures the idea that people are not held responsible for situations over which they had no control. Or, to put it positively, people are held responsible for situations in as much as they were/are able to influence them. To give an example, if the ticket holder in our introductory situation chose to flip a coin to allocate the concert ticket to one of his friends, the friends's kindness perceptions of the ticket holder's choice would depend on his procedural choice even after the realization of the move of chance. He would not be held responsible for the realization itself as he was not able to influence it after he had taken the decision to flip a coin. Similarly, 'ex ante' the ticket holder's kindness perception of his own choice is also based only on what he is able to influence, i.e. he does not hold himself responsible for the realization of the flip of the coin but only for his procedural choice. To capture this idea we define the decision context of a person $i$ in any history $h_{i}^{x}$. In every history $h_{i}^{x}$ the decision context comprises, first, all passed procedural choices on the path to history $h_{i}^{x},(\omega)_{h_{i}^{x}}$, with $(\omega)_{h_{i}^{x}}=\left\{\left(\omega_{i}\right)_{h_{i}^{x}}, \ldots,\left(\omega_{N}\right)_{h_{i}^{x}}\right\}$. Remember, the knowledge of all passed procedural choices on the path to history $h_{i}^{x}$ is included in the updated procedural strategies $m_{i}\left(h_{i}^{x}\right)$ and the updated first order beliefs $b_{i j}\left(h_{i}^{x}\right)$. Second, the decision context includes the realizations of the moves of chance on the path up to history $h_{i}^{x},(a)_{h_{i}^{x}}$, and, third, the remaining explicit probability distributions, $(\mathcal{P})_{\neg h_{i}^{x}}$, where $\neg h_{i}^{x}$ indicates all histories beside the histories on the path up to $h_{i}^{x}$. Hence, formally speaking:

\footnotetext{
${ }^{2}$ For a more detailed discussion of this issue refer to Dufwenberg and Kirchsteiger (2004).
} 
Definition 3 The decision context in any history $h_{i}^{x}$ is a tuple:

$$
\left\langle(\omega)_{h_{i}^{x}},(a)_{h_{i}^{x}},(\mathcal{P})_{\neg h_{i}^{x}}\right\rangle .
$$

This means it is the collection of $i)$ all passed procedural choices of all players on the path to $h_{i}^{x},(\omega)_{h_{i}^{x}}$, ii) all passed realizations of the moves of chance on the path up to $h_{i}^{x},(a)_{h_{i}^{x}}$, and iii) the unreached explicit probability distributions, $(\mathcal{P})_{\neg h_{i}^{x}}$.

Intuitively speaking the decision context can be understood as the 'informational background' which players use to evaluate their own kindness towards others and, hence, to take their decisions. It is also the 'informational background' which is used by other players in later stages to evaluate the kindness of passed choices by others. More precisely, the decision context helps to decide in how far others were consciously aiming at a certain decision, i.e. pure action, or whether it was by chance that it was chosen.

We can now capture the idea that players strive to be kind if treated kindly and are unkind if treated unkindly by assuming that every player $i \in \mathcal{N} \backslash\{0\}$ chooses a behavioral procedural strategy, $m_{i}$, that maximizes his utility defined as:

$$
U_{i}=\pi_{i}+\sum_{j \neq i} Y_{i j} \cdot\left(\kappa_{i j} \cdot \lambda_{i j i}\right)
$$

where $i, j \in \mathcal{N} \backslash\{0\}, \kappa_{i j}$ is the believed kindness of player $i$ to player $j$ and $\lambda_{i j i}$ is player $i$ 's belief about the kindness of player $j$ towards himself.

More precisely, player $i$ 's utility is the sum of $N$ terms. The first term $\pi_{i}$ represents player $i$ 's self interest. It is his expected material payoff in any history $h_{i}^{x}$ after which he moves. It obviously depends on his own behavioral procedural strategy, $m_{i}\left(h_{i}^{x}\right)$, his belief about the others' behavioral procedural strategies, $b_{i j}\left(h_{i}^{x}\right), \forall j \neq i$, all past outcomes/realizations of procedures $(a)_{h_{i}^{x}}$ until history $h_{i}^{x}$, and, finally, on the explicit probability distributions in all histories that have not been reached yet during the course of the game, $(\mathcal{P})_{\neg h^{x}}$. Hence:

$$
\pi_{i}=\pi_{i}\left(m_{i}\left(h_{i}^{x}\right),\left(b_{i j}\left(h_{i}^{x}\right)\right)_{j \neq i},(a)_{h_{i}^{x}},(\mathcal{P})_{\neg h^{x}}\right) .
$$

It can easily be seen that, as we allow for explicit randomizations in our class of procedural games our definition of expected material payoffs differs from the definition by Dufwenberg and Kirchsteiger (2005). It takes the player $i$ 's decision context in history $h_{i}^{x}$ into account.

The following $N-1$ terms, $\sum_{j \neq i} Y_{i j} \cdot\left(\kappa_{i j} \cdot \lambda_{i j i}\right)$, in equation (2), on the other hand, represent player $i$ 's reciprocity payoff with respect to each other player $j \neq i$. The factor $Y_{i j}$ is a non-negative reciprocity parameter which describes player $i$ 's sensitivity to the (un)kindness of player $j$. The higher $Y_{i j}$ the more sensitive to reciprocity player $i$ is. Finally the factors $\kappa_{i j}$ and $\lambda_{i j i}$ capture respectively the kindness of player $i$ to any other player $j$ and player $i$ 's perceived kindness of player $j$ towards him. Intuitively, kindness $\kappa_{i j}$ is positive or negative depending on whether $i$ is kind or unkind to $j$ and perceived kindness $\lambda_{i j i}$ is positive (negative) if player $i$ beliefs player $j$ to be kind (unkind) to him. Notice, reciprocity is captured by the factorial specification of the kindness parameters, $\kappa_{i j}$ and $\lambda_{i j i}$. It drives players to match perceived kindness (positive $\lambda_{i j i}$ ) with kindness (positive $\kappa_{i j}$ ) and perceived unkindness (negative $\lambda_{i j i}$ ) with unkindness (negative $\kappa_{i j}$ ).

This brings us to the formal definition of kindness, $\kappa_{i j}$ : 
Definition 4 The kindness of player $i$ to another player $j \neq i$ at any history $h_{i}^{x} \in \mathcal{H}$ is given by the function $\kappa_{i j}: \mathcal{M}_{i} \times \Pi_{j \neq i} \mathcal{B}_{i j} \rightarrow \Re$ defined as:

$$
\kappa_{i j}=\pi_{j}\left(m_{i}\left(h_{i}^{x}\right),\left(b_{i j}\left(h_{i}^{x}\right)\right)_{j \neq i},(a)_{h_{i}^{x}},(\mathcal{P})_{\neg h^{x}}\right)-\pi_{j}^{e_{i}}\left(\left(b_{i j}\left(h_{i}^{x}\right)\right)_{j \neq i},(a)_{h_{i}^{x}},(\mathcal{P})_{\neg h^{x}}\right) .
$$

The kindness of player $i$ towards player $j$ in history $h_{i}^{x}$ is defined as the difference between the expected material payoff of player $j, \pi_{j}$, that player $i$ intends to give $j$ and the average expected material payoff, $\pi_{j}^{e_{i}}\left(\left(b_{i j}\left(h_{i}^{x}\right)\right)_{j \neq i},(a)_{h_{i}^{x}},(\mathcal{P})_{\neg h^{x}}\right)$, defined as:

$$
\begin{aligned}
& \pi_{j}^{e_{i}}\left(\left(b_{i j}\left(h_{i}^{x}\right)\right)_{j \neq i},(a)_{h_{i}^{x}},(\mathcal{P})_{\neg h^{x}}\right) \\
= & \frac{1}{2}\left[\max \left\{\pi_{j}\left(m_{i}\left(h_{i}^{x}\right),\left(b_{i j}\left(h_{i}^{x}\right)\right)_{j \neq i},(a)_{h_{i}^{x}},(\mathcal{P})_{\neg h^{x}}\right) \mid m_{i}\left(h_{i}^{x}\right) \in \mathcal{M}_{i}\right\}\right. \\
& \left.+\min \left\{\pi_{j}\left(m_{i}\left(h_{i}^{x}\right),\left(b_{i j}\left(h_{i}^{x}\right)\right)_{j \neq i},(a)_{h_{i}^{x}},(\mathcal{P})_{\neg h^{x}}\right) \mid m_{i}\left(h_{i}^{x}\right) \in \mathcal{E}_{i}\right\}\right] .
\end{aligned}
$$

Think of $\pi_{j}^{e_{i}}$ as a norm for $i$ describing the 'equitable' payoff for player $j$ when $i$ 's beliefs about the other players' behavior are summarized by $\left(b_{i j}\left(h_{i}^{x}\right)\right)_{j \neq i}$, the passed realization on the path to $h_{i}^{x}$ are $(a)_{h_{i}^{x}}$ and the unreached explicit probability distributions are given by $(\mathcal{P})_{\neg h^{x}}$. Thus, when $\pi_{j}^{e_{i}{ }^{2}}=\pi_{j}$ then player $i$ 's kindness towards player $j$ is zero. Intuitively the above definition means that player $i$ is kinder the more he expects to give player $j$ relative to the average that he could give him given his beliefs about the other players play. To exemplify consider, for example, history $h_{2}^{5}$ of game $\Gamma_{3}$. The behavioral procedural strategy of player $2, m_{2}\left(h_{2}^{5}\right)$, as well as his first-order belief over the profile of player $1, b_{21}\left(h_{2}^{5}\right)$, and the passed realized move of nature, $(a)_{h_{2}^{5}}=\{(L)\}$, define history $h_{2}^{5}$. Furthermore, player 2's behavioral procedural strategy together with his first-order belief and the remaining probability distributions, $(\mathcal{P})_{\neg h^{x}}$, on the other hand, define what player 2 is willing to give to player 1 in expected terms as well as what he could give him. Assume, for example, that player 2's behavioral procedural strategy in $h_{2}^{5}$ is $m_{2}\left(h_{2}^{5}\right)=\left(\omega\left(h_{2}^{4}\right), \omega\left(h_{2}^{5}\right), \omega\left(h_{2}^{6}\right), \omega\left(h_{2}^{7}\right)\right)$. It can easily be seen that player 2 intends to give player $1 \pi_{1}\left(h_{2}^{5}\right)=1800$, i.e. according to $m_{2}\left(h_{2}^{5}\right)$ he will choose $\omega\left(h_{2}^{5}\right)$ after his history $h_{2}^{5}$. On the other hand, the average of the maximum and minimum which he could give to player 1 is $\pi_{1}^{e_{2}}\left(h_{2}^{5}\right)=\frac{1}{2}(1800)+\frac{1}{2}(0)=900$. Hence, player 2's kindness towards player 1 in $h_{2}^{5}$ is:

$$
\begin{aligned}
\kappa_{21}\left(h_{2}^{5}\right) & =\pi_{1}\left(h_{2}^{5}\right)-\pi_{1}^{e_{2}}\left(h_{2}^{5}\right)=1800-900 \\
& =900 .
\end{aligned}
$$

The above definition of kindness is a necessary adaptation from Dufwenberg and Kirchsteiger (2004) in the context of our procedural game. It includes the decision context on which players base their decisions.

The definition of perceived kindness, $\lambda_{i j i}$, also requires a change though. As said above, in the evaluation of intentions agents take into account in how far others were/are actually responsible for the unraveled play. Hence, it would be unreasonable to assume that player 2 in game $\Gamma_{3}$ perceived the kindness of player 1 in histories $h_{2}^{5}$ and $h_{2}^{6}$ differently. It is simply by chance that either of the two histories are reached. In order to capture this we assume that players always evaluate the other players' kindness on the basis of the decision context 
in which the others have taken their last procedural choice. Remember, a decision context characterizes the 'informational base' on which a decision is taken. As players know all passed procedural choices as well as the realizations of moves of chance along the path up to $h_{i}^{x}$, they obviously not only know their own current decision context, but they can also deduce all passed decision contexts which were the basis of the other players' last procedural choices. Denote the history in which any player $j \neq i$ has made his last procedural choice along the path up to $h_{i}^{x}$ as $h_{i}^{x}\left(h_{j}^{l}\right)$. When player $i$ evaluates the kindness of player $j$ 's procedural choice in history $h_{i}^{x}$, he, hence, uses player $j$ 's decision context in $h_{i}^{x}\left(h_{j}^{l}\right)$ :

$$
\left\langle(\omega)_{h_{i}^{x}\left(h_{j}^{l}\right)},(a)_{h_{i}^{x}\left(h_{j}^{l}\right)},(\mathcal{P})_{\neg h_{i}^{x}\left(h_{j}^{l}\right)}\right\rangle,
$$

where $(\omega)_{h_{i}^{x}\left(h_{j}^{l}\right)}$ defines all passed procedural choices on the path to $h_{i}^{x}$ up to history $h_{j}^{l}$, $(a)_{h_{i}^{x}\left(h_{j}^{l}\right)}$ defines all passed realizations of moves of chance on the path to history $h_{i}^{x}$ up to history $h_{j}^{l}$ and $(\mathcal{P})_{\neg h_{i}^{x}\left(h_{j}^{l}\right)}$ indicates all remaining explicit randomizations in $h_{j}^{l}$. Evaluating player $j$ 's kindness only on the basis of the decision context in which he has made his last procedural choice on the path up to history $h_{i}^{x}$ ensures that player $j$ is held solely responsible for the decisions that he has explicitly taken himself. To exemplify, in both histories $h_{2}^{5}$ and $h_{2}^{6}$ player 2 evaluates player 1's kindness on the basis of player 1's decision context at the history, $h_{1}^{0}$ :

$$
\left\langle(\omega)_{h_{2}^{5}\left(h_{1}^{0}\right)},(a)_{h_{2}^{5}\left(h_{1}^{0}\right)},(\mathcal{P})_{\neg h_{2}^{5}\left(h_{1}^{0}\right)}\right\rangle=\left\langle(\omega)_{h_{2}^{6}\left(h_{1}^{0}\right)},(a)_{h_{2}^{6}\left(h_{1}^{0}\right)},(\mathcal{P})_{\neg h_{2}^{6}\left(h_{1}^{0}\right)}\right\rangle,
$$

in which player 1 had to take his last procedural decision, i.e. $h_{j}^{l}=h_{1}^{0}$. In other words, in histories $h_{2}^{5}$ and $h_{2}^{6}$ player 2 does not take the realization of the move of chance after history $h_{0}^{2}$ into account when evaluating the kindness of player 1 . The realization of the move of chance after $h_{0}^{2}$ is by chance and hence not the responsibility of player 1 .

Given this let perceived kindness be defined as:

Definition 5 Player $i$ 's beliefs about how kind player $j \neq i$ is to $i$ at history $h_{i}^{x} \in \mathcal{H}$ is given by the function $\lambda_{i j i}: \mathcal{B}_{i j} \times \Pi_{i \neq j} \mathcal{C}_{i j i} \rightarrow \Re$ defined as:

$$
\begin{aligned}
\lambda_{i j i}= & \pi_{i}\left(b_{i j}\left(h_{i}^{x}\right),\left(c_{i j q}\left(h_{i}^{x}\right)\right)_{q \neq j},(a)_{h_{i}^{x}\left(h_{j}^{l}\right)},(\mathcal{P})_{\neg h_{i}^{x}\left(h_{j}^{l}\right)}\right) \\
& -\pi_{i}^{e j}\left(\left(c_{i j i}\left(h_{i}^{x}\right)\right)_{i \neq j},(a)_{h_{i}^{x}\left(h_{j}^{l}\right)},(\mathcal{P})_{\neg h_{i}^{x}\left(h_{j}^{l}\right)}\right),
\end{aligned}
$$

where $h_{i}^{x}\left(h_{j}^{l}\right)$ is the last history after which player $j$ has moved on the path to $h_{i}^{x}$.

As one can see, similar to the definition of kindness also perceived kindness is defined as the difference between what player $i$ beliefs to receive in expected material payoff relative to the average that he could have gotten. To exemplify, assume now again that players find themselves in history $h_{2}^{5}$ of game $\Gamma_{3}$. We have seen above that, given player 2's updated behavioral procedural strategy, his first-order belief and the past realizations of the moves of chance up to history $h_{2}^{5}$, player 2's kindness towards player 1 is 900 in $h_{2}^{5}$. In addition to player 2's updated first-order belief $b_{21}\left(h_{2}^{5}\right)=\left(\omega^{\prime}\left(h_{1}^{0}\right)\right)$, let now player 2's updated second order 
belief be $c_{212}\left(h_{2}^{5}\right)=\left(\omega\left(h_{2}^{4}\right), \omega\left(h_{2}^{5}\right), \omega\left(h_{2}^{6}\right), \omega\left(h_{2}^{7}\right)\right)$. The kindness that player 2 perceives from player 1 is then given by:

$$
\begin{aligned}
\lambda_{212}\left(h_{2}^{5}\right)= & \pi_{2}\left(b_{21}\left(h_{2}^{5}\right), c_{212}\left(h_{2}^{5}\right),(a)_{h_{2}^{5}\left(h_{1}^{0}\right)},(\mathcal{P})_{\neg h_{2}^{5}\left(h_{1}^{0}\right)}\right) \\
& -\pi_{2}^{e_{1}}\left(c_{212}\left(h_{2}^{5}\right),(a)_{h_{2}^{5}\left(h_{1}^{0}\right)},(\mathcal{P})_{\neg h_{2}^{5}\left(h_{1}^{0}\right)}\right) \\
= & \left(\frac{1}{2}(1800)+\frac{1}{2}(200)\right)-\frac{1}{2}((1800)+(200)) \\
= & 0 .
\end{aligned}
$$

This means, player 2 has the impression in history $h_{2}^{5}$ that player 1 intends to give him $\pi_{2}\left(h_{2}^{5}\right)=1000$. As 1000 is also the 'equitable' payoff that player 1 could have given to him, player 2 judges player 1's kindness to be 0 . Now consider history $h_{2}^{4}$, on the other hand, which is the starting point of an identical subgame. Player 2's perceived kindness of player 1's behavioral procedural strategy given his updated beliefs, $b_{21}\left(h_{2}^{4}\right)=\left(\omega_{1}\left(h_{1}^{0}\right)\right)$ and $c_{212}\left(h_{2}^{4}\right)=\left(\omega\left(h_{2}^{4}\right), \omega\left(h_{2}^{5}\right), \omega\left(h_{2}^{6}\right), \omega\left(h_{2}^{7}\right)\right)$ is:

$$
\begin{aligned}
\lambda_{212}\left(h_{2}^{4}\right)= & \pi_{2}\left(b_{21}\left(h_{2}^{4}\right), c_{212}\left(h_{2}^{4}\right),(a)_{h_{2}^{4}\left(h_{1}^{0}\right)},(\mathcal{P})_{\neg h_{2}^{4}\left(h_{1}^{0}\right)}\right) \\
& -\pi_{2}^{e_{1}}\left(c_{212}\left(h_{2}^{4}\right),(a)_{h_{2}^{4}\left(h_{1}^{0}\right)},(\mathcal{P})_{\neg h_{2}^{4}\left(h_{1}^{0}\right)}\right) \\
= & (200)-\frac{1}{2}((1800)+(200)) \\
= & -800 .
\end{aligned}
$$

Hence, although $h_{2}^{4}$ and $h_{2}^{5}$ are starting points of identical subgames, players perceives the situations totally different, i.e. perceived kindness of 0 in $h_{2}^{5}$ vs. perceived kindness of -800 in $h_{2}^{4}$. It follows that as both histories are perceived differently, optimal reactions in one history might not be optimal in the other even though the subsequent situation seems to be the same. This exemplifies that reciprocal agents do care about the way a certain situation has come about or, in other words, reciprocity inherently leads to procedural concerns.

This completes the description of the reciprocal preferences in the context of our procedural game. Putting together the procedural game, $\Gamma$, as defined in (1) and the vector of utilities, $\left(U_{i}\right)_{i \in \mathcal{N} \backslash\{0\}}$, as defined in (2) we get a tuple

$$
\Gamma^{p}=\left\langle\Gamma,\left(U_{i}\right)_{i \in \mathcal{N} \backslash\{0\}}\right\rangle .
$$

We refer to $\Gamma^{p}$ as a procedural game with reciprocity preferences. Note, as the 'psychological game with reciprocity preferences' defined by Dufwenberg and Kirchsteiger (2004) $\Gamma^{p}$ is not a 'traditional game'. In line with Dufwenberg and Kirchsteiger (2004), utility functions, $U_{i}$, are defined on richer domains including subjective beliefs. Different to them, however, and also different to 'traditional games' agents in our setting choose for procedures, as defined in Definition (1), rather than actions and strategies.

As a solution concept for our class of procedural games with reciprocity preferences we propose the sequential reciprocity equilibrium (SRE) defined by Dufwenberg and Kirchsteiger (2004). This means, each player in each history chooses his optimal procedure given his 
beliefs. The players' initial first and second order beliefs are required to be correct, and following each history of play the beliefs are updated as explained above.

Let $\mathcal{M}_{i}\left(h_{i}^{x}, m\right)$ be the non-empty set of behavioral procedural strategies that prescribe, for each player $i \in \mathcal{N} \backslash\{0\}$, the same choices as the strategy $m_{i}\left(h_{i}^{x}\right)$ for all histories other than $h_{i}^{x}$. Given this, the sequential reciprocity equilibrium (SRE) in the context of our procedural game with reciprocity preferences is defined as:

Definition 6 The profile $m^{*}=\left(m_{i}^{*}\right)_{i \in \mathcal{N} \backslash\{0\}}$ is a sequential reciprocity equilibrium (SRE) if for all $i \in \mathcal{N} \backslash\{0\}$ and for each history $h_{i}^{x} \in \mathcal{H}$ it holds that

1. $m_{i}^{*}\left(h_{i}^{x}\right) \in$

$\arg \max _{m_{i} \in \mathcal{M}_{i}\left(h_{i}^{x}, m\right)} U_{i}\left(m_{i}\left(h_{i}^{x}\right),\left(b_{i j}\left(h_{i}^{x}\right),\left(c_{i j q}\left(h_{i}^{x}\right)\right)_{q \neq j}\right)_{j \neq i},(a)_{h_{i}^{x}},(\mathcal{P})_{\neg h_{i}^{x}}\right)$,

2. $b_{i j}=m_{j}^{*}$ for all $j \neq i$,

3. $c_{i j q}=m_{q}^{*}$ for all $j \neq i, q \neq j$.

Condition 1 assures that a SRE is a strategy profile such that at history $h_{i}^{x}$ player $i$ makes choices which maximize his utility given his beliefs and given that he follows his equilibrium strategy at other histories. At the initial stage, conditions (2) and (3) guarantee that the initial beliefs are correct. At any subsequent history, condition (1) requires that beliefs assign probability one to the sequence of choices that define that history, but are otherwise as the initial beliefs.

Concluding, in this section we have formally defined the motivation of reciprocal agents in the context of our procedural game and have given a glimpse of the impact of procedural choices on the strategic interaction of reciprocal agents. In the following section we will more fully analyze the impact of procedural choices by applying the concept of the sequential reciprocity equilibrium to two examples.

\section{Applications}

The first application is the 'Sequential Prisoners Dilemma' also analyzed by Dufwenberg and Kirchsteiger (2004). The second is the 'So Long, Sucker' game in the spirit of Nalebuff and Shubik (1988) and Dufwenberg and Kirchsteiger (2004). Note, a full description of the strategic interaction and all possible equilibria that might arise in these two situations is beyond the scope of this paper. We, therefore, limit the analysis to the characterization of only one equilibrium to demonstrate the impact and importance of procedural concerns. Results and intuitions are presented in this section, mathematical proofs are relegated to the Appendix.

\section{Example 1: Sequential Prisoners Dilemma}

Consider the Sequential Prisoners Dilemma in Figure $4:^{1}$ 


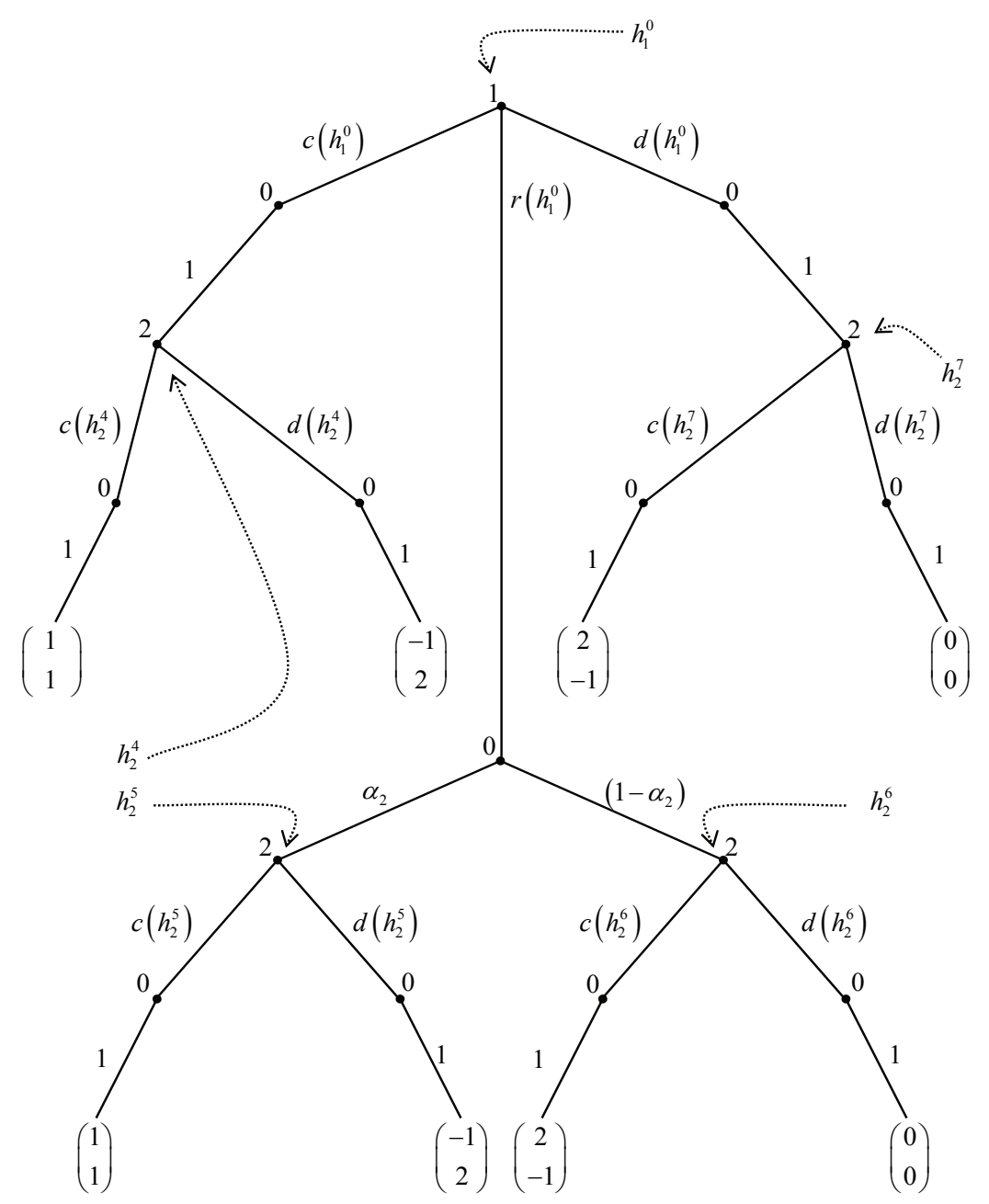

Figure 4: Game $\Gamma_{4}$

As can easily be seen, game $\Gamma_{4}$ is an adaptation of the sequential prisoners dilemma analyzed by Dufwenberg and Kirchsteiger (2004). The difference is that in $\Gamma_{4}$ player 1 cannot only choose to cooperate $(c)$ and defect $(d)$, but can also explicitly randomize by choosing procedure $(r)$. One sequential reciprocity equilibrium is:

Result 1 If player 1's and 2's sensitivity to reciprocity, $Y_{1}$ and $Y_{2}$, is such that

$$
0<Y_{1}<\frac{1}{2}
$$

and

$$
Y_{2}>\frac{1}{4 \alpha_{2}-3}
$$

and player 1's procedure $r\left(h_{1}^{0}\right)$ is associated with an explicit probability distribution such that $1>\alpha_{2}>\frac{3}{4}$, then the SRE is given by player 1 choosing $r\left(h_{1}^{0}\right)$ in history $h_{1}^{0}$ and player 2 
choosing c $\left(h_{2}^{4}\right), c\left(h_{2}^{5}\right), c\left(h_{2}^{6}\right)$ and $d\left(h_{2}^{7}\right)$ in histories $h_{2}^{4}, h_{2}^{5}, h_{2}^{6}$ and $h_{2}^{7}$ respectively. ${ }^{3}$

Proof: see Appendix.

The intuition is the following. If $\alpha_{2}$ is such that $1>\alpha_{2}>\frac{3}{4}$, player 2 perceives player 1's procedural choice as kind. If, in addition, his sensitivity to reciprocity $Y_{2}$ is high enough, i.e. $Y_{2}>\frac{1}{4 \alpha_{2}-3}$, then he reciprocates player 1's kindness by choosing $(c)$ in history $h_{2}^{6}$. At the same time player 2 punishes player 1 in equilibrium at history $h_{2}^{7}$ which is the starting point of a payoff equivalent subgame. The difference between histories $h_{2}^{6}$ and $h_{2}^{7}$ is that the explicit probability $\alpha_{2}$ is such that player 2 perceives player 1's choice of $(r)$ as kind. He does not attribute enough responsibility for the outcome, i.e. history $h_{2}^{6}$, to player 1 to make it worth while to punish him. Furthermore, since $Y_{1}$ is relatively small, player 1 is mainly interested by money and his expected monetary payoff is highest by playing $(r)$ given that player 2 does not play $(d)$ following player 1's choice of $(r)$.

Dufwenberg and Kirchsteiger (2004), on the other hand, showed in the context of their setting that if player 2's sensitivity to reciprocity is strong enough, he cooperates if player 1 cooperates and defects if player 1 defects. Furthermore, they showed that if player 1's sensitivity to reciprocity is low and player 2's sensitivity is high, cooperation is player 1's equilibrium behavior for both monetary and reciprocity reasons.

Comparing the results shows that their equilibrium predictions are very sensitive to the availability of other procedures to take the same decision. As player 1 can also use procedure $(r)$ to take his decision cooperation is no longer his optimal action given that player 2 is very sensitive to reciprocity. He chooses $(r)$ because this makes player 2 to cooperate even in history $h_{2}^{6}$ which is identical to the subgame starting in $h_{2}^{7}$. Hence, procedural choices influence the kindness and perceived kindness of players and therefore influence the interaction of reciprocal agents.

\section{Example 2: The 'So Long, Sucker' Game}

In the following we will apply the concept of the sequential reciprocity equilibrium to the example, $\Gamma_{5}$, in Figure 5. ${ }^{1}$ Example $\Gamma_{5}$ is an adaptation of the 'So Long, Sucker' game also analyzed by Nalebuff and Shubik (1988) and Dufwenberg and Kirchsteiger (2004). With $\varepsilon=0, \Gamma_{5}$ is a strategic situation in which player 1 has to decide on whom of two other players to give a zero payoff. Following his decision, the player who was unfavorably treated is called upon to decide whether player 1 should get 3 or whether both the others should equally get a payoff of 1 . Intuitively it looks as if player 1 is 'a priori' worst off, as whoever he chooses will feel badly treated, and hence take revenge on player 1 by giving him the lowest possible monetary payoff.

\footnotetext{
${ }^{3}$ For simplicity we denote the sensitivity of reciprocity as $Y_{i}$ in example 1 . In example 2 we stick to $Y_{i j}$ as defined in equation (2) to avoid confusion.
} 


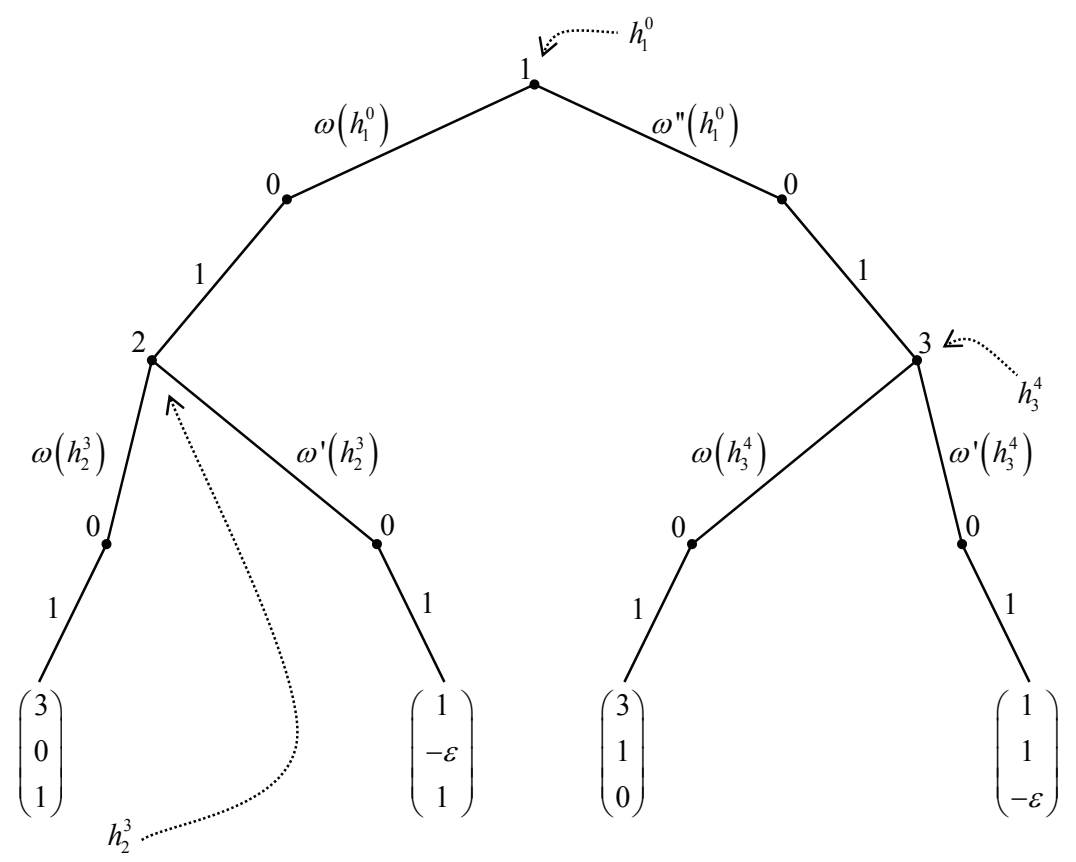

Figure 5: Game $\Gamma_{6}$

However, if all players are solely motivated by purely selfish monetary concerns, this outcome is not guaranteed, as players 2 and 3 are indifferent between all their choices given that $\varepsilon=0$. In order to allow for the possibility of revenge, Nalebuff and Shubik (1988) depart from the usual selfishness assumption, and assume that the players have lexicographically ordered objectives. This means that each player primarily maximizes his monetary payoff, but in case some choices yield exactly the same monetary payoff ties are broken so as to allow a player to take revenge. Dufwenberg and Kirchsteiger (2004), on the other hand, show that if agents behave reciprocally this outcome is also guaranteed for $\varepsilon \geq 0$. More precisely, they show that for any $\varepsilon \geq 0$ there exist sensitivities to reciprocity $Y_{21}>0$ and $Y_{31}>0$ for which taking revenge on player 1 is the best alternative for player 2 and 3 . As said above, if players 2 and 3 are willing to take revenge even if it is costly, it seems that player 1 is trapped, as whatever he does, his action is perceived unkind by the player who has to take the subsequent decision.

As in the Sequential Prisoners Dilemma also in this application these results crucially depend on the fact that players 2 and 3 attribute full intentionality to player 1 . In other words, Nalebuff and Shubik (1988)'s and Dufwenberg and Kirchsteiger (2004)'s result is contingent on the unavailability of other procedures for player 1 to resolve the conflict between him and the other players. Consider game $\Gamma_{6}$ in Figure 6 : 


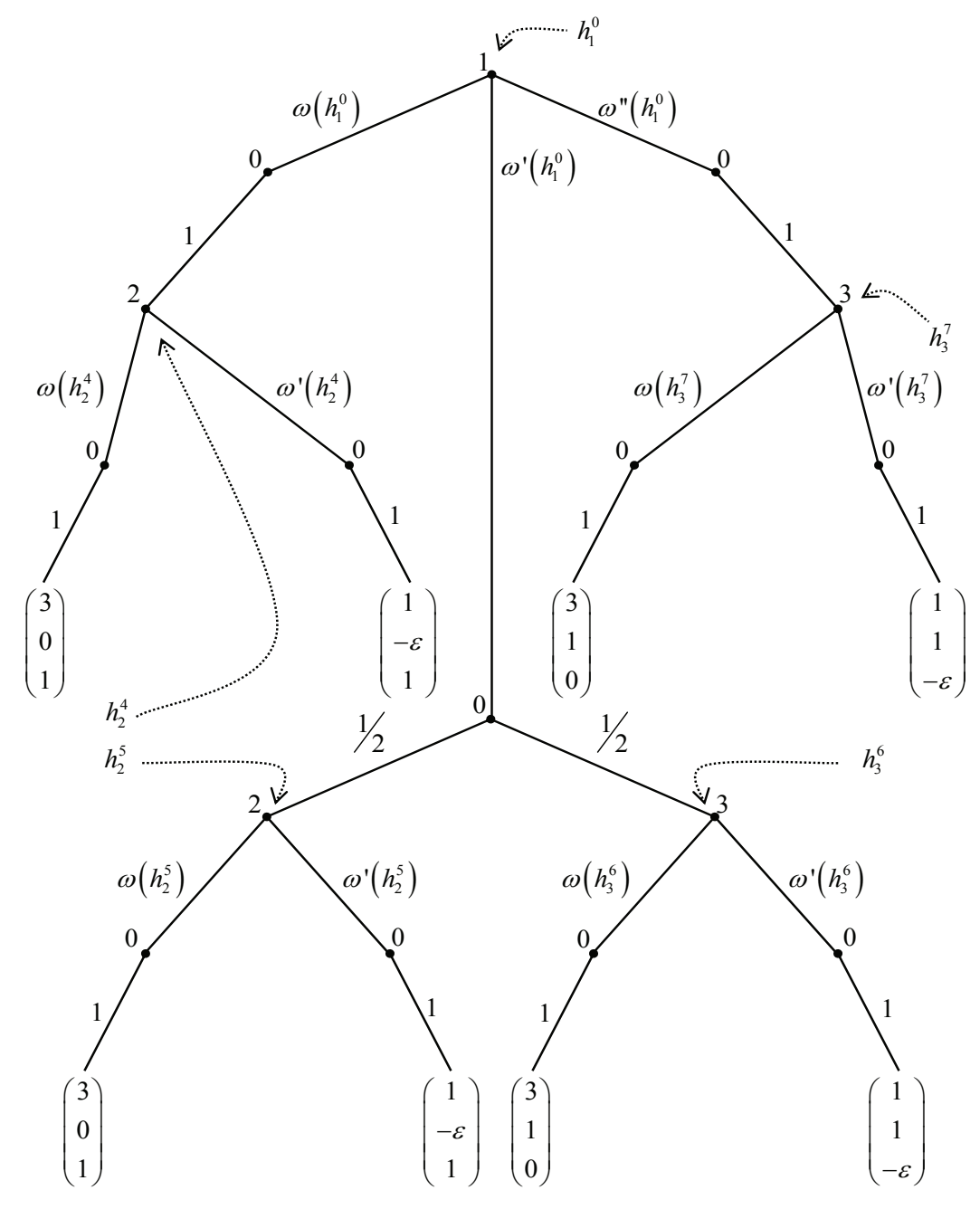

Figure 6: Game $\Gamma_{6}$

As can easily be seen, the only difference between games $\Gamma_{5}$ and $\Gamma_{6}$ lies in the fact that in the latter player 1 cannot only take his decision directly but can also e.g. flip a coin, i.e. choose $\omega_{1}^{\prime}\left(h_{1}^{0}\right)$, to take it. Hence, he has an additional procedure which he can use to take his decision. It can be shown that with the help of this procedure player 1 can avoid the conflict with the others. More precisely:

Result 2 If player 1, 2 and 3 have a sensitivity to reciprocity of

$$
\begin{gathered}
Y_{12}=Y_{13} \geq 0, \\
Y_{21} \geq \frac{\varepsilon}{\varepsilon+1}
\end{gathered}
$$

and

$$
Y_{31} \geq \frac{\varepsilon}{\varepsilon+1},
$$


then the only equilibrium is given by players 2 and 3 playing

$$
\left(\omega_{2}^{\prime}\left(h_{2}^{4}\right), \omega_{2}\left(h_{2}^{5}\right)\right)
$$

and

$$
\left(\omega_{3}^{\prime}\left(h_{3}^{6}\right), \omega_{2}\left(h_{3}^{7}\right)\right)
$$

respectively and player 1 choosing $\omega_{1}^{\prime}\left(h_{1}^{0}\right)$.

This means, if players 2 and 3 are enough sensitive to reciprocity, they will punish player 1 , if he chooses one of them directly, and will be kind to him, if he chooses to take the decision by e.g. 'flipping a coin'. Knowing this, player 1 will choose to flip a coin, given that his sensitivity to reciprocity is equal for players 2 and 3, as this gives him a higher monetary as well as reciprocity payoff. In other words, by choosing e.g. to flip a coin, player 1 can get out of his 'trap'. Players 2 and 3 respectively perceive player 1's procedural strategy $\omega_{1}\left(h_{1}^{0}\right), \omega_{1}^{\prime \prime}\left(h_{1}^{0}\right)$ as unkind and $\omega_{1}^{\prime}\left(h_{1}^{0}\right)$ as kind. If player 1 chooses e.g. to flip a coin, they do not attribute the outcome of the randomization to player 1 , as he is only responsible for choosing the procedure but not for the outcome itself. Player 1, on the other hand, chooses $\omega_{1}^{\prime}\left(h_{1}^{0}\right)$ for monetary as well as reciprocity reasons.

This highlights ones more how procedural choices influence the strategic interaction of reciprocal agents.

\section{Conclusion}

As we have seen, any decision in human interactions is inherently associated with a procedure which characterizes the way in which the decision is taken. This means it is impossible to take a decision without deciding on how to take it. It is widely accepted in other scientific disciplines and it has been shown experimentally that people react differently to identical outcomes depending on the procedures which have led to them. Hence, people are concerned about the way in which decisions are taken. Nevertheless economic theory has so far neglected the impact of procedural choices on human interaction. It has ignored procedural concerns as traditional economic theory is based on consequentialist preferences. However, if preferences are solely outcome oriented, it can hardly be explained why people should react differently to 'outcomewise' identical situations which only differ in the procedures which have led to them.

Only in recent years theories of reciprocity have contested the consequentialist view in economic theory by assuming that agents also receive a psychological payoff which, broadly speaking, depends on the agents' perceived intentions of others. As said before, when people behave reciprocally they evaluate the intentions of others and reciprocate kind with kind and unkind with unkind behavior. The evaluation of intentions is implicitly connected to the assignment of responsibilities for outcomes. The assignment of responsibilities, in turn, is related to the amount of control that people have over outcomes. It has been shown in our paper that procedural choices influence the control that people have over outcomes and, hence, influence the attribution of responsibilities and the evaluation of intentions. Dufwenberg and Kirchsteiger (2004)'s theory of sequential reciprocity captures situations in 
which agents have full control over outcomes and, hence, are held fully responsible for all consequences of their actions. In contrast to this, in our class of procedural games agents can choose between different procedures, which differ in the probabilities that they assign to outcomes. Given this we show, in line with attribution theory, that the less influence people have on outcomes the less responsibility and intentionality is attributed to them.

By defining a class of procedural games we have been able to distinguishing between procedures which are used to take decisions and the decisions themselves. Furthermore, assuming reciprocal agents and defining the decision context as the 'informational background' which any decision is based upon, we have demonstrated that procedural concerns are actually an inherent feature of any interaction of reciprocal agents.

\section{List of References}

1. Anderson, R. A. and Otto, A. L. (2003), Perceptions of fairness in the justice system: A cross-cultural comparison, Social Behavior and Personality. 31, 557-564.

2. Battigalli, P. and Dufwenberg, M. (2005), Dynamic Psychological Games, Mimeo. IGIER-Bocconi

3. Bies, R. J. and Tyler, T. R. (1993), The "litigation mentality" in organizations: A test of alternative psychological explanations, Organization Science, 4, 352-366.

4. Blader, S. L. and Tyler, T. R. (2003), A four-component model of procedural justice: Defining the meaning of a "fair" process, Personality and Social Psychology Bulletin, $29,747-758$.

5. Blount, S. (1995), When Social Outcomes Aren't Fair: The Effect of Casual Attributions on Preferences, Organizational Behavior and Human Decision Processes, 63, 131-144.

6. Bolton, G. and Ockenfels, A. (2000), ERC: A Theory of Equity, Reciprocity, and Competition, American Economic Review, 90(1), 166-193.

7. Bolton, G., Brandts, J. and Ockenfels, A. (2005), Fair Procedures: Evidence from Games Involving Lotteries, Economic Journal, 115 (506), 1054-1076.

8. Collie, T., Bradley, G. and Sparks, B. A. (2002), Fair process revisited: Differential effects of interactional and procedural justice in the presence of social comparison information, Journal of Experimental Social Psychology, 38, 545-555.

9. Dufwenberg, M. and Kirchsteiger, G. (2004), A Theory of Sequential Reciprocity, Games and Economic Behavior, 47 (2), 268-298.

10. De Cremer, D. and van Knippenberg, D. (2003), Cooperation with leaders in social dilemmas: On the effects of procedural fairness and outcome favorability in structural cooperation, Organizational Behavior and Human Decision Processes, 91, 1-11. 
11. Falk, A. and Fischbacher, U. (2006), A Theory of Reciprocity, Games and Economic Behavior 54 (2), 293-315.

12. Fehr, E. and Schmidt, K. (1999), A Theory Of Fairness, Competition, And Cooperation, The Quarterly Journal of Economics, 114(3), 817-868.

13. Geanakoplos, J., Pearce, D. and Stacchetti, E. (1989), Psychological Games and Sequential Rationality, Games and Economic Behavior, 1, 60-79.

14. Heider, F. (1958), The Psychology of Interpersonal Relations, New York: John Wiley \& Sons.

15. Kelley, H. H. (1967), Attribution in social psychology, Nebraska Symposium on Motivation, 15, 192-238.

16. Kelley, H. H. (1973), The processes of causal attribution, American Psychologist, 28, 107-128.

17. Konovsky, M. A. (2000), Understanding Procedural Justice and Its Impact on Business Organizations, Journal of Management, 26 (3), 489-511.

18. Krawczyk, M. (2007), A model of procedural and distributive fairness, Mimeo. University of Amsterdam.

19. Lemons, M. A. and Jones, C.A. (2001), Procedural justice in promotion decisions: using perceptions of fairness to build employee commitment, Journal of Managerial Psychology, 16 (4), 268-281.

20. Lind, E. A. and Tyler, T. R. (1988), The social psychology of procedural justice, New York, NY, US: Plenum Press.

21. Lind, E. A., Greenberg, J., Scott, K. S. and Welchans, T. D. (2000), The winding road from employee to complainant: Situational and psychological determinants of wrongful termination claims, Administrative Science Quarterly, 45, 557-590.

22. Murphy, K. (2004), The role of trust in nurturing compliance: A study of accused tax avoiders, Law and Human Behavior, 28, 187-209.

23. Nalebuff, B. and Shubik, M. (1988), Revenge And Rational Play, Papers 138, Princeton, Woodrow Wilson School - Public and International Affairs.

24. Thibaut J. and Walker, L. (1975), Procedural Justice, Erlbaum, Hillsdale, NJ.

25. Trautmann, S. T. (2006), A Fehr-Schmidt Model for Process Fairness, Working Paper, CREED, University of Amsterdam.

26. Tyler, T. R. (1990), Why people obey the law, New Haven, CT, US: Yale University Press. 
27. Tyler, T. R. (2003), Procedural justice, legitimacy, and the effective rule of law, in Tonry, M. (ed.), Crime and justice: A review of research, 30, 283-358.

28. Rabin, M. (1993), Incorporating Fairness into Game Theory and Economics, American Economic Review, 83 (5), 1281-1302.

29. Roberts, K. and Markel, K. S. (2001), Claiming in the name of fairness: Organizational justice and the decision to file for workplace injury compensation, Journal of Occupational Health Psychology, 6, 332-347.

30. Ross, M. and Fletcher, G. J. O. (1985), Attribution and Social Perception, in G. Lindsey $\&$ E. Aronson (eds.), The Handbook of Social Psychology, 2, 73-114.

31. Wenzel, M. (2002) The impact of outcome orientation and justice concerns on tax compliance: The role of taxpayers' identity, Journal of Applied Psychology, 87, 629645.

\section{Appendix}

\section{Proof to result (1):}

In this proof we show under what conditions the behavior as defined in Result (1) is the equilibrium behavior. Note, as defined in Definition (6) we assume that players' beliefs are correct. Given this, we analyze under what conditions they can be sustained in equilibrium. It can easily be seen that if player 2's second order belief about player 1's belief is $\left(c\left(h_{2}^{4}\right), c\left(h_{2}^{5}\right), c\left(h_{2}^{6}\right), d\left(h_{2}^{7}\right)\right)$, then player 2's believed equitable payoff is $\pi_{2}^{e_{1}}=\frac{1}{2}(1)+\frac{1}{2}(0)=$ $\frac{1}{2}$. Hence, player 2's perceived kindness if player 1 chooses $c\left(h_{1}^{0}\right)$ is

$$
\lambda_{212}\left(h_{2}^{4}\right)=1-\frac{1}{2}=\frac{1}{2},
$$

where 1 is player 2's expected monetary payoff and $\frac{1}{2}$ his equitable payoff given his second order belief.

Secondly, if player 1 plays $r\left(h_{1}^{0}\right)$ player 2's perceived kindness of player 1's procedural choice is

$$
\begin{aligned}
\lambda_{212}\left(h_{2}^{5}\right) & =\lambda_{212}\left(h_{2}^{6}\right) \\
& =\alpha_{2}(1)+\left(1-\alpha_{2}\right)(-1)-\frac{1}{2} \\
& =2 \alpha_{2}-\frac{3}{2},
\end{aligned}
$$

and, thirdly, if player 1 plays $d\left(h_{1}^{0}\right)$, it is

$$
\lambda_{212}\left(h_{2}^{7}\right)=0-\frac{1}{2}=-\frac{1}{2} .
$$

From equation (4) it can directly be seen that player 2 perceives player 1's procedural choice $r\left(h_{1}^{0}\right)$ as kind or unkind depending on $\alpha_{2}$. If $\alpha_{2}>\frac{3}{4}$ then player 1's choice of $r\left(h_{1}^{0}\right)$ is perceived as kind. Therefore, 
Remark 1 If $\alpha_{2}$ is such that

$$
1>\alpha_{2}>\frac{3}{4}
$$

then player 2 perceives player 1's procedural choice $r\left(h_{1}^{0}\right)$ as kind.

Henceforth we assume that player 1's procedure $r\left(h_{1}^{0}\right)$ is associated with an explicit probability distribution $\alpha_{2}>\frac{3}{4}$.

We said before that player 1's first order belief is $\left(c\left(h_{2}^{4}\right), c\left(h_{2}^{5}\right), c\left(h_{2}^{6}\right), d\left(h_{2}^{7}\right)\right)$. Furthermore, we said that in equilibrium this belief has to be correct. Hence, under what condition do we expect player 2 to choose $c\left(h_{2}^{4}\right)$ following player 1's choice of $c\left(h_{1}^{0}\right)$ ? By playing $c\left(h_{2}^{4}\right)$ player 2 receives the following utility

$$
u_{2}\left(c\left(h_{2}^{4}\right)\right)=1+Y_{2}(1)\left(\frac{1}{2}\right)
$$

where $\kappa_{21}\left(c\left(h_{2}^{4}\right)\right)=1-\frac{1}{2}((1)+(-1))=1$ is player 2's kindness to player 1 by playing $c\left(h_{2}^{4}\right)$. On the other hand, by playing $d\left(h_{2}^{4}\right)$ player 2 's utility is

$$
u_{2}\left(d\left(h_{2}^{4}\right)\right)=2+Y_{2}(-1)\left(\frac{1}{2}\right)
$$

where $\kappa_{21}\left(d\left(h_{2}^{4}\right)\right)=-1-\frac{1}{2}((1)+(-1))=-1$. Hence player 2 plays $c\left(h_{2}^{4}\right)$ in history $h_{2}^{4}$ if

$$
1+Y_{2}(1)\left(\frac{1}{2}\right) \geq 2+Y_{2}(-1)\left(\frac{1}{2}\right)
$$

This reduces to

$$
Y_{2} \geq 1
$$

This shows that if player 1 plays $c\left(h_{1}^{0}\right)$, player 2 plays $c\left(h_{2}^{4}\right)$ if $Y_{2} \geq 1$.

Remark 2 If player 1 plays $c\left(h_{1}^{0}\right)$, player 2 plays $c\left(h_{2}^{4}\right)$ if $Y_{2} \geq 1$.

Going back to player 1's first order belief, under what conditions do we expect player 2 to choose $d\left(h_{2}^{7}\right)$ following player 1's choice of $d\left(h_{1}^{0}\right)$ ?

In history $h_{2}^{7}$ it is easy to see that player 2 's monetary and reciprocity payoff induce him to chooses $d\left(h_{2}^{7}\right)$ for all $Y_{2} \geq 0$. Hence, if player 1 plays $d\left(h_{1}^{0}\right)$, player 2 plays $d\left(h_{2}^{7}\right)$ if $Y_{2} \geq 0$.

Remark 3 If player 1 plays $d\left(h_{1}^{0}\right)$, player 2 plays d $\left(h_{2}^{4}\right)$ if $Y_{2} \geq 0$.

Finally, under what conditions do we expect player 2 to choose $c\left(h_{2}^{5}\right)$ in $h_{2}^{5}$ and $c\left(h_{2}^{6}\right)$ in $h_{2}^{6}$ following player 1's choice of $r\left(h_{1}^{0}\right)$ ?

Assume that player 1 has chosen $r\left(h_{1}^{0}\right)$. Doing the analogous calculations as above for player 2's behavior in history $h_{2}^{5}$ one can see that player 2 plays $c\left(h_{2}^{5}\right)$ in $h_{2}^{5}$ if

$$
1+Y_{2}(1)\left(2 \alpha_{2}-\frac{3}{2}\right) \geq 2+Y_{2}(-1)\left(2 \alpha_{2}-\frac{3}{2}\right)
$$


where the lhs is $u_{2}\left(c\left(h_{2}^{5}\right)\right)$ and the $r h s$ is $u_{2}\left(d\left(h_{2}^{5}\right)\right)$. The above reduces to

$$
Y_{2} \geq \frac{1}{4 \alpha_{2}-3}
$$

Note, as $\alpha_{2}>\frac{3}{4}$ we know that $Y_{2} \geq \frac{1}{4 \alpha_{2}-3}>1$. This shows that any player 2 with $Y_{2} \geq \frac{1}{4 \alpha_{2}-3}$ would play $c\left(h_{2}^{4}\right)$ in history $h_{2}^{4}$ and $c\left(h_{2}^{5}\right)$ in history $h_{2}^{5}$. Finally, in history $h_{2}^{6}$ the analogous calculations as in $h_{2}^{5}$ and $h_{2}^{4}$ are

$$
-1+Y_{2}(1)\left(2 \alpha_{2}-\frac{3}{2}\right) \geq 0+Y_{2}(-1)\left(2 \alpha_{2}-\frac{3}{2}\right)
$$

where the lhs is $u_{2}\left(c\left(h_{2}^{6}\right)\right)$ and the rhs is $u_{2}\left(d\left(h_{2}^{6}\right)\right)$. The above also reduces to

$$
Y_{2} \geq \frac{1}{4 \alpha_{2}-3}
$$

Hence, also here it holds that if $Y_{2} \geq \frac{1}{4 \alpha_{2}-3}$ player 2 plays $c\left(h_{2}^{6}\right)$ in history $h_{2}^{6}$.

Remark 4 If player 1 plays $r\left(h_{1}^{0}\right)$, player 2 plays $c\left(h_{2}^{5}\right)$ in $h_{2}^{5}$ and $c\left(h_{2}^{6}\right)$ in $h_{2}^{6}$ if $Y_{2} \geq \frac{1}{4 \alpha_{2}-3}$.

Concluding, as we have seen above, if $Y_{2} \geq \frac{1}{4 \alpha_{2}-3}$, it holds that player 2' equilibrium behavior is characterized by $c\left(h_{2}^{4}\right), c\left(h_{2}^{5}\right), c\left(h_{2}^{6}\right)$ and $d\left(h_{2}^{7}\right)$ in histories $h_{2}^{4}, h_{2}^{5}, h_{2}^{6}$ and $h_{2}^{7}$ respectively.

Let us now turn to player 1. Player 1's perceived kindness of player 2's equilibrium procedural strategy is

$$
\begin{aligned}
\lambda_{121}\left(h_{1}^{0}\right) & =\left(q+2 q^{\prime}-q^{\prime} \alpha_{2}\right)-\left(1-q^{\prime} \alpha_{2}-q\right) \\
& =2 q+2 q^{\prime}-1,
\end{aligned}
$$

where $q$ and $q^{\prime}$ are player 1's second order beliefs associated with his procedures $c\left(h_{1}^{0}\right)$ and $r\left(h_{1}^{0}\right)$. His kindness to player 2 , on the other hand, is

$$
\kappa_{12}\left(c\left(h_{1}^{0}\right)\right)=1-\frac{1}{2}=\frac{1}{2},
$$

by playing $c\left(h_{1}^{0}\right)$,

$$
\begin{aligned}
\kappa_{12}\left(r\left(h_{1}^{0}\right)\right) & =\alpha_{2}-(1-\alpha)-\frac{1}{2} \\
& =2 \alpha_{2}-\frac{3}{2},
\end{aligned}
$$

by playing $r\left(h_{1}^{0}\right)$ and

$$
\kappa_{12}\left(c\left(h_{1}^{0}\right)\right)=0-\frac{1}{2}=-\frac{1}{2},
$$

by playing $d\left(h_{1}^{0}\right)$. 
Putting the pieces together one can see that player 1 chooses $r\left(h_{1}^{0}\right)$ in equilibrium if for $q^{\prime}=1$ and $q=0$ two conditions hold: $\left.i\right) u_{1}\left(r\left(h_{1}^{0}\right)\right) \geq u_{1}\left(c\left(h_{1}^{0}\right)\right)$ and $\left.i i\right) u_{1}\left(r\left(h_{1}^{0}\right)\right) \geq$ $u_{1}\left(d\left(h_{1}^{0}\right)\right)$. The first condition boils down to

$$
\left(2-\alpha_{2}\right)+Y_{1}\left(2 \alpha_{2}-\frac{3}{2}\right) \geq(1)+Y_{1}\left(\frac{1}{2}\right)
$$

which reduces to

$$
Y_{1} \leq \frac{1}{2}
$$

The second condition furthermore boils down to

$$
\left(2-\alpha_{2}\right)+Y_{1}\left(2 \alpha_{2}-\frac{3}{2}\right) \geq(0)+Y_{1}\left(-\frac{1}{2}\right),
$$

which holds for all $Y_{1} \geq 0$. Hence, given player 2's behavior, the equilibrium behavior of player 1 is characterized by $r\left(h_{1}^{0}\right)$ if $0<Y_{1} \leq \frac{1}{2}$.

Remark 5 Given player 2's equilibrium behavior $\left(c\left(h_{2}^{4}\right), c\left(h_{2}^{5}\right), c\left(h_{2}^{6}\right), d\left(h_{2}^{7}\right)\right)$, player 1 plays $r\left(h_{1}^{0}\right)$ if $0<Y_{1} \leq \frac{1}{2}$.

In other words, if player 2's sensitivity to reciprocity is high and player 1's is not too strong, the equilibrium behavior for both players is player 1 choosing the procedure $r\left(h_{1}^{0}\right)$ and player 2 choosing $\left(c\left(h_{2}^{5}\right), c\left(h_{2}^{6}\right)\right)$ in response. This concludes the proof of Result (1).

\section{Proof to result (2):}

In analogy to the aforementioned proof, we first show under what conditions $\left(\omega_{2}^{\prime}\left(h_{2}^{4}\right), \omega_{2}\left(h_{2}^{5}\right)\right)$ and $\left(\omega_{3}^{\prime}\left(h_{3}^{6}\right), \omega_{2}\left(h_{3}^{7}\right)\right)$ simultaneously represent the equilibrium behavior of players 2 and 3 . Then, secondly, we show the conditions for which it is best for player 1 to choose $\omega_{1}^{\prime}\left(h_{1}^{0}\right)$, given the behavior of players 2 and 3 .

If $\left(\omega_{2}^{\prime}\left(h_{2}^{4}\right), \omega_{2}\left(h_{2}^{5}\right)\right)$ and $\left(\omega_{3}^{\prime}\left(h_{3}^{6}\right), \omega_{2}\left(h_{3}^{7}\right)\right)$ are player 2's and 3's procedural strategies, then the most and least that player 1 can give to player 2 and 3 is either 1 or $-\varepsilon$. Hence, it can easily be seen that the perceived kindness of player 2 and 3 in either of the four histories $h_{2}^{4}$, $h_{2}^{5}, h_{3}^{6}, h_{2}^{7}$ is:

$$
\begin{aligned}
\lambda_{212}\left(h_{2}^{4}\right) & =\lambda_{313}\left(h_{3}^{7}\right) \\
& =-\varepsilon-\frac{1}{2}(1-\varepsilon) \\
& =-\frac{1}{2}(1+\varepsilon), \\
\lambda_{212}\left(h_{2}^{5}\right)= & \lambda_{212}\left(h_{3}^{6}\right)=\lambda_{313}\left(h_{2}^{5}\right)=\lambda_{313}\left(h_{3}^{6}\right) \\
= & \frac{1}{2}-\frac{1}{2}(1-\varepsilon) \\
& =\frac{1}{2} \varepsilon,
\end{aligned}
$$


and

$$
\begin{aligned}
\lambda_{212}\left(h_{3}^{7}\right) & =\lambda_{313}\left(h_{2}^{4}\right) \\
& =1-\frac{1}{2}(1-\varepsilon) \\
& =\frac{1}{2}(1+\varepsilon),
\end{aligned}
$$

where $\pi_{2}^{e_{1}}=\pi_{3}^{e_{1}}=\frac{1}{2}(1-\varepsilon)$. In other words, if player 1 chooses $\omega_{1}\left(h_{1}^{0}\right)$ player 2 perceives this as unkind and player 3 as kind. On the other hand, if player 1 chooses $\omega_{1}^{\prime \prime}\left(h_{1}^{0}\right)$, player 2 perceives this as kind and player 3 as unkind. Furthermore, if player 1 takes his decision by flipping a coin, i.e. $\omega_{1}^{\prime}\left(h_{1}^{0}\right)$, then both players do not perceive this as unkind as $\varepsilon \geq 0$.

Remark 6 Player 2 perceives player 1's procedural choice of $\omega_{1}\left(h_{1}^{0}\right)$ as unkind. Likewise, player 3 perceives player 1's procedural choice $\omega_{1}^{\prime \prime}\left(h_{1}^{0}\right)$ as unkind. On the other hand, both player do not perceive player 1 's choice $\omega_{1}^{\prime}\left(h_{1}^{0}\right)$ as unkind.

Consider now all histories in turn. Looking at history $h_{2}^{4}$ after which player 2 has to choose one can see that player 2 can either show a kindness of

$$
\begin{aligned}
\kappa_{12}\left(\omega_{2}\left(h_{2}^{4}\right)\right) & =3-\frac{1}{2}(3+1) \\
& =1
\end{aligned}
$$

by playing $\omega_{2}\left(h_{2}^{4}\right)$ or he can show a kindness of

$$
\begin{aligned}
\kappa_{12}\left(\omega_{2}^{\prime}\left(h_{2}^{4}\right)\right) & =1-\frac{1}{2}(3+1) \\
& =-1
\end{aligned}
$$

by playing $\omega_{2}^{\prime}\left(h_{2}^{4}\right)$. Obviously, player 2's behavior in history $h_{2}^{4}$ in general also creates some (un)kindness towards player 3. In our case, however, 3's monetary payoff is invariant to player 2's choice in $h_{2}^{4}$. Hence, player 2's kindness towards player 3 is 0 in $h_{2}^{4}$. Given this, the utilities from either of player 2's choices are

$$
u_{2}\left(\omega_{2}\left(h_{2}^{4}\right)\right)=(0)+Y_{21}(1)\left(-\frac{1}{2}(1+\varepsilon)\right),
$$

and

$$
u_{2}\left(\omega_{2}^{\prime}\left(h_{2}^{4}\right)\right)=(-\varepsilon)+Y_{21}(-1)\left(-\frac{1}{2}(1+\varepsilon)\right) .
$$

Again in equilibrium player 2 chooses the latter if $u_{2}\left(\omega_{2}^{\prime}\left(h_{2}^{4}\right)\right) \geq u_{2}\left(\omega_{2}\left(h_{2}^{4}\right)\right)$. This can be written as

$$
(-\varepsilon)+Y_{21}(-1)\left(-\frac{1}{2}(1+\varepsilon)\right) \geq(0)+Y_{21}(1)\left(-\frac{1}{2}(1+\varepsilon)\right),
$$

which reduces to

$$
Y_{21} \geq \frac{\varepsilon}{\varepsilon+1}
$$


This means if $Y_{21} \geq \frac{\varepsilon}{\varepsilon+1}$ then player 2 takes revenge on player 1 by choosing $\omega_{2}^{\prime}\left(h_{2}^{4}\right)$ in history $h_{2}^{4}$. From the symmetry of the game it necessarily also follows that everything which holds for player 2 in history $h_{2}^{4}$ also holds for player 3 in history $h_{3}^{7}$. In other words if

$$
Y_{31} \geq \frac{\varepsilon}{\varepsilon+1}
$$

then player 3 takes revenge on player 1 in history $h_{3}^{7}$ by playing $\omega_{3}^{\prime}\left(h_{3}^{7}\right)$.

Remark 7 Players 2 and 3 take revenge on player 1 by playing $\omega_{2}^{\prime}\left(h_{2}^{4}\right)$ in $h_{2}^{4}$ and $\omega_{3}^{\prime}\left(h_{3}^{7}\right)$ in $h_{3}^{7}$ respectively, if $Y_{21}, Y_{31} \geq \frac{\varepsilon}{\varepsilon+1}$.

Turning now to histories $h_{2}^{5}$ and $h_{2}^{6}$ one can see that due to the symmetry of the situation both players, 2 and 3, perceive player 1's kindness identically. Therefore, in history $h_{2}^{5}$ player 2 's utilities from choosing either of his procedures is

$$
u_{2}\left(\omega_{2}\left(h_{2}^{5}\right)\right)=(0)+Y_{21}(1)\left(\frac{1}{2} \varepsilon\right)
$$

and

$$
u_{2}\left(\omega_{2}^{\prime}\left(h_{2}^{5}\right)\right)=(-\varepsilon)+Y_{21}(-1)\left(\frac{1}{2} \varepsilon\right) .
$$

He chooses $\omega_{2}\left(h_{2}^{5}\right)$ rather than $\omega_{2}^{\prime}\left(h_{2}^{5}\right)$ if $u_{2}\left(\omega_{2}\left(h_{2}^{5}\right)\right) \geq u_{2}\left(\omega_{2}^{\prime}\left(h_{2}^{5}\right)\right)$, i.e.

$$
(0)+Y_{21}(1)\left(\frac{1}{2} \varepsilon\right) \geq(-\varepsilon)+Y_{21}(-1)\left(\frac{1}{2} \varepsilon\right) \text {, }
$$

which reduces to

$$
Y_{21} \geq-1
$$

Note, this holds for all $Y_{21} \geq 0$. Again, for equal reasons also player 3 chooses $\omega_{3}\left(h_{3}^{6}\right)$ rather than $\omega_{2}^{\prime}\left(h_{3}^{6}\right)$ in history $h_{3}^{6}$ if $Y_{21} \geq 0$.

Remark 8 If player 2's and 3's sensitivity to reciprocity is

$$
Y_{21} \geq 0
$$

and

$$
Y_{31} \geq 0
$$

then they respectively choose $\omega_{2}\left(h_{2}^{5}\right)$ and $\omega_{3}\left(h_{3}^{6}\right)$ in histories $h_{2}^{5}$ and $h_{3}^{6}$ following player 1 's choice of $\omega_{1}^{\prime}\left(h_{1}^{0}\right)$.

Concluding, if $Y_{21} \geq \frac{\varepsilon}{\varepsilon+1}$ and $Y_{31} \geq \frac{\varepsilon}{\varepsilon+1}$ then players 2 and 3 play $\left(\omega_{2}^{\prime}\left(h_{2}^{4}\right), \omega_{2}\left(h_{2}^{5}\right)\right)$ and $\left(\omega_{3}^{\prime}\left(h_{3}^{6}\right), \omega_{2}\left(h_{3}^{7}\right)\right)$ in their histories $h_{2}^{4}, h_{2}^{5}$ and $h_{3}^{6}, h_{3}^{7}$ respectively.

Given this under what conditions is it best for player 1 to choose $\omega_{1}^{\prime}\left(h_{1}^{0}\right)$ ? Assume for simplicity that player 1's sensitivity to reciprocity is equal towards both, player 2 and 3 . In other words, assume that $Y_{12}=Y_{13}=Y$. Denote player 1's second order beliefs about player 2's and 3's beliefs $p_{2}, p_{2}^{\prime}$ and $\left(1-p_{2}-p_{2}^{\prime}\right)$ as well as $p_{3}, p_{3}^{\prime}$ and $\left(1-p_{3}-p_{3}^{\prime}\right)$. More precisely, 
let $p_{i}$ and $p_{i}^{\prime}$ be player 1 's belief about the probabilities that any player $i \in\{2,3\}$ attaches to player 1's procedures $\omega_{1}\left(h_{1}^{0}\right)$ and $\omega_{1}^{\prime}\left(h_{1}^{0}\right)$ respectively. Therefore, player 1's perceived kindness from player 2's and 3's procedural strategies is

$$
\begin{aligned}
\lambda_{121} & =p_{2}(-1)+p_{2}^{\prime}\left(\frac{1}{2}(1)+\frac{1}{2}(0)\right)+\left(1-p_{2}-p_{2}^{\prime}\right)(0) \\
& =p_{2}^{\prime}\left(\frac{1}{2}\right)-p_{2}
\end{aligned}
$$

and

$$
\begin{aligned}
\lambda_{131} & =p_{3}(0)+p_{3}^{\prime}\left(\frac{1}{2}(0)+\frac{1}{2}(1)\right)+\left(1-p_{3}-p_{3}^{\prime}\right)(-1) \\
& =p_{3}^{\prime}\left(\frac{1}{2}\right)-\left(1-p_{3}-p_{3}^{\prime}\right)
\end{aligned}
$$

Player 1's kindness, on the other hand, towards player 2 and 3 is given by $\kappa_{12}\left(\omega_{1}\left(h_{1}^{0}\right)\right)=$ $\kappa_{13}\left(\omega_{1}^{\prime \prime}\left(h_{1}^{0}\right)\right)=-\frac{1}{2}(1+\varepsilon), \kappa_{13}\left(\omega_{1}\left(h_{1}^{0}\right)\right)=\kappa_{12}\left(\omega_{1}^{\prime \prime}\left(h_{1}^{0}\right)\right)=\frac{1}{2}(1+\varepsilon)$ and $\kappa_{12}\left(\omega_{1}^{\prime}\left(h_{1}^{0}\right)\right)=$ $\kappa_{13}\left(\omega_{1}^{\prime}\left(h_{1}^{0}\right)\right)=\frac{1}{2} \varepsilon$.

Hence, given that players 2 and 3 choose $\left(\omega_{2}^{\prime}\left(h_{2}^{4}\right), \omega_{2}\left(h_{2}^{5}\right)\right)$ and $\left(\omega_{3}^{\prime}\left(h_{3}^{6}\right), \omega_{2}\left(h_{3}^{7}\right)\right)$ the utilities from all of player 1's procedural choices can be written as

$$
\begin{aligned}
u_{1}\left(\omega_{1}\left(h_{1}^{0}\right)\right)= & 1+Y\left(-\frac{1}{2}(1+\varepsilon)\right)\left(p_{2}^{\prime}\left(\frac{1}{2}\right)-p_{2}\right) \\
& +Y\left(\frac{1}{2}(1+\varepsilon)\right)\left(p_{3}^{\prime}\left(\frac{1}{2}\right)-\left(1-p_{3}-p_{3}^{\prime}\right)\right)
\end{aligned}
$$

by playing $\omega_{1}\left(h_{1}^{0}\right)$,

$$
\begin{aligned}
u_{1}\left(\omega_{1}^{\prime}\left(h_{1}^{0}\right)\right)= & 3+Y\left(\frac{1}{2} \varepsilon\right)\left(p_{2}^{\prime}\left(\frac{1}{2}\right)-p_{2}\right) \\
& +Y\left(\frac{1}{2} \varepsilon\right)\left(p_{3}^{\prime}\left(\frac{1}{2}\right)-\left(1-p_{3}-p_{3}^{\prime}\right)\right)
\end{aligned}
$$

by playing $\omega_{1}^{\prime}\left(h_{1}^{0}\right)$ and

$$
\begin{aligned}
u_{1}\left(\omega_{1}^{\prime \prime}\left(h_{1}^{0}\right)\right)= & 1+Y\left(\frac{1}{2}(1+\varepsilon)\right)\left(p_{2}^{\prime}\left(\frac{1}{2}\right)-p_{2}\right) \\
& +Y\left(-\frac{1}{2}(1+\varepsilon)\right)\left(p_{3}^{\prime}\left(\frac{1}{2}\right)-\left(1-p_{3}-p_{3}^{\prime}\right)\right)
\end{aligned}
$$

by playing $\omega_{1}^{\prime \prime}\left(h_{1}^{0}\right)$.

Obviously, player 1 plays $\omega_{1}^{\prime}\left(h_{1}^{0}\right)$ if $u_{1}\left(\omega_{1}^{\prime}\left(h_{1}^{0}\right)\right) \geq u_{1}\left(\omega_{1}\left(h_{1}^{0}\right)\right)$ and $u_{1}\left(\omega_{1}^{\prime}\left(h_{1}^{0}\right)\right) \geq u_{1}\left(\omega_{1}^{\prime \prime}\left(h_{1}^{0}\right)\right)$ with $p_{2}=p_{3}=0, p_{2}^{\prime}=p_{3}^{\prime}=1$ and $p_{2}^{\prime \prime}=p_{3}^{\prime \prime}=0$. The first of the two conditions can be written as

$$
\begin{aligned}
& 3+Y\left(\frac{1}{2} \varepsilon\right)\left(\frac{1}{2}\right)+Y\left(\frac{1}{2} \varepsilon\right)\left(\frac{1}{2}\right) \\
\geq & 1-Y\left(\frac{1}{2}(1+\varepsilon)\right)\left(\frac{1}{2}\right)+Y\left(\frac{1}{2}(1+\varepsilon)\right)\left(\frac{1}{2}\right),
\end{aligned}
$$


which holds for all $Y>0$. Secondly, it has to hold that

$$
\begin{aligned}
& 3+Y\left(\frac{1}{2} \varepsilon\right)\left(\frac{1}{2}\right)+Y\left(\frac{1}{2} \varepsilon\right)\left(\frac{1}{2}\right) \\
\geq & 1+Y\left(\frac{1}{2}(1+\varepsilon)\right)\left(\frac{1}{2}\right)-Y\left(\frac{1}{2}(1+\varepsilon)\right)\left(\frac{1}{2}\right),
\end{aligned}
$$

which is identical to the above. Hence, whenever $Y=Y_{12}=Y_{13}>0$ it holds that player 1's best response to player 2's and 3's procedural strategy $\left(\omega_{2}^{\prime}\left(h_{2}^{4}\right), \omega_{2}\left(h_{2}^{5}\right)\right)$ and $\left(\omega_{3}^{\prime}\left(h_{3}^{6}\right), \omega_{2}\left(h_{3}^{7}\right)\right)$ is to play $\omega_{1}^{\prime}\left(h_{1}^{0}\right)$.

Remark 9 Given player 2's and 3's equilibrium play, player 1 chooses procedure $\omega_{1}^{\prime}\left(h_{1}^{0}\right)$, if $Y=Y_{12}=Y_{13}>0$.

This concludes the proof of Result (2). 